\title{
Supporting Information: Polarized Fock States and the Dynamical Casimir Effect in Molecular Cavity Quantum Electrodynamics
}

\author{
Arkajit Mandal,* Sebastian Montillo Vega, and Pengfei Huo* \\ Department of Chemistry, University of Rochester, Rochester, NY, 14627 \\ E-mail: amandal4@ur.rochester.edu; pengfei.huo@rochester.edu
}

Molecular Hamiltonian in the Adiabatic Representation

The time-independent Schrödinger equation for to a molecular system is given as

$$
\hat{H}_{\mathrm{M}}|\Psi(\mathbf{r}, \mathbf{R})\rangle=\left[\hat{T}_{\mathbf{R}}+\hat{H}_{\mathrm{el}}\right]|\Psi(\mathbf{R}, \mathbf{r})\rangle=\mathcal{E}|\Psi(\mathbf{r}, \mathbf{R})\rangle
$$

We use the Born-Huang expansion of $\Psi(\mathbf{r}, \mathbf{R})$ in Eqn. S1,

$$
|\Psi(\mathbf{r}, \mathbf{R})\rangle=\sum_{i}\left|\psi_{i}(\mathbf{r}, \mathbf{R})\right\rangle \otimes\left|\varphi_{i}(\mathbf{R})\right\rangle
$$

where $\left|\varphi_{i}(\mathbf{R})\right\rangle$ is the nuclear wavefunction, and $\left|\psi_{i}(\mathbf{r}, \mathbf{R})\right\rangle$ is the adiabatic electronic wavefunction. Projecting $\left\langle\psi_{j}(\mathbf{r}, \mathbf{R})\right|$ on Eqn. S1 we obtain,

$$
\begin{aligned}
\mathcal{E}\left|\chi_{j}(\mathbf{R})\right\rangle & =\left\langle\psi_{j}(\mathbf{r}, \mathbf{R})\left|\hat{H}_{\mathrm{M}}\right| \Psi(\mathbf{R}, \mathbf{r})\right\rangle=\left\langle\psi_{j}(\mathbf{r}, \mathbf{R})\left|\hat{H}_{\mathrm{M}} \sum_{i}\right| \psi_{i}(\mathbf{r}, \mathbf{R})\right\rangle \otimes\left|\varphi_{i}(\mathbf{R})\right\rangle \\
& =\sum_{i}\left[\left\langle\psi_{j}(\mathbf{r}, \mathbf{R})\left|\hat{T}_{\mathrm{R}}\right| \psi_{i}(\mathbf{r}, \mathbf{R})\right\rangle+\left\langle\psi_{j}(\mathbf{r}, \mathbf{R})\left|\hat{H}_{\mathrm{el}}\right| \psi_{i}(\mathbf{r}, \mathbf{R})\right\rangle\right]\left|\varphi_{i}(\mathbf{R})\right\rangle
\end{aligned}
$$

Using the fact that $\left\{\left|\psi_{i}(\mathbf{r}, \mathbf{R})\right\rangle\right\}$ are the adiabatic states such that they are the eigenstates of the electronic Hamiltonian, the matrix elements of $\hat{H}_{\mathrm{el}}$ are $\left\langle\psi_{j}(\mathbf{r}, \mathbf{R})\left|\hat{H}_{\mathrm{el}}\right| \psi_{i}(\mathbf{r}, \mathbf{R})\right\rangle=E_{i} \delta_{i j}$. 
Further, we obtain the matrix elements of the nuclear Kinetic energy operator as follows

$$
\begin{aligned}
& \left\langle\psi_{j}(\mathbf{r}, \mathbf{R})\left|\hat{\mathbf{T}}_{\mathbf{R}}\right| \psi_{i}(\mathbf{r}, \mathbf{R})\right\rangle \\
& =-\frac{\hbar^{2}}{2 M}\left[\left\langle\psi_{j}(\mathbf{r}, \mathbf{R}) \mid \psi_{i}(\mathbf{r}, \mathbf{R})\right\rangle \nabla_{\mathbf{R}}^{2}+2\left\langle\psi_{j}(\mathbf{r}, \mathbf{R})\left|\nabla_{\mathbf{R}}\right| \psi_{i}(\mathbf{r}, \mathbf{R})\right\rangle \nabla_{R}+\left\langle\psi_{j}(\mathbf{r}, \mathbf{R})\left|\nabla_{\mathbf{R}}^{2}\right| \psi_{i}(\mathbf{r}, \mathbf{R})\right\rangle\right] .
\end{aligned}
$$

Using the fact that $\left\langle\psi_{j}(\mathbf{r}, \mathbf{R}) \mid \psi_{i}(\mathbf{r}, \mathbf{R})\right\rangle=\delta_{i j}$, as well as the following definitions of derivative coupling and the $\mathrm{BO}$ correction

$$
\begin{aligned}
\mathbf{d}_{i j}(\mathbf{R}) & =\left\langle\psi_{j}(\mathbf{r}, \mathbf{R})\left|\nabla_{\mathbf{R}}\right| \psi_{i}(\mathbf{r}, \mathbf{R})\right\rangle \\
\mathbf{D}_{i j}(\mathbf{R}) & =\left\langle\psi_{j}(\mathbf{r}, \mathbf{R})\left|\nabla_{\mathbf{R}}^{2}\right| \psi_{i}(\mathbf{r}, \mathbf{R})\right\rangle,
\end{aligned}
$$

we can write the nuclear Kinetic energy operator as follows

$$
\begin{aligned}
& \hat{\mathbf{T}}_{\mathbf{R}}=\sum_{i j}\left\langle\psi_{j}(\mathbf{r}, \mathbf{R})\left|\hat{\mathbf{T}}_{R}\right| \psi_{i}(\mathbf{r}, \mathbf{R})\right\rangle\left|\psi_{i}(\mathbf{r}, \mathbf{R})\right\rangle\left\langle\psi_{j}(\mathbf{r}, \mathbf{R})\right| \\
& =-\frac{\hbar^{2}}{2 M} \sum_{i j}\left[\nabla_{\mathbf{R}}^{2} \delta_{i j}+2 \mathbf{d}_{i j}(\mathbf{R}) \cdot \nabla_{\mathbf{R}}+\mathbf{D}_{i j}(\mathbf{R})\right]\left|\psi_{i}(\mathbf{r}, \mathbf{R})\right\rangle\left\langle\psi_{j}(\mathbf{r}, \mathbf{R})\right| \\
& =-\frac{\hbar^{2}}{2 M}\left[\nabla_{\mathbf{R}}^{2} \otimes \hat{\mathbb{1}}_{\mathbf{e}}+2 \mathbf{d} \cdot \nabla_{\mathbf{R}}+\mathbf{D}\right]
\end{aligned}
$$

where $\hat{\mathbb{1}}_{\mathrm{e}}$ is the identity operator in the electronic subspace, $\mathbf{d}$ and $\mathbf{D}$ are the matix form of $\mathbf{d}_{i j}$ and $\mathbf{D}_{i j}$.

Finally, D can be expressed as

$$
\begin{aligned}
\mathbf{D} & =\sum_{i j}\left\langle\psi_{j}(\mathbf{r}, \mathbf{R})\left|\nabla_{\mathbf{R}}^{2}\right| \psi_{i}(\mathbf{r}, \mathbf{R})\right\rangle\left|\psi_{i}(\mathbf{r}, \mathbf{R})\right\rangle\left\langle\psi_{j}(\mathbf{r}, \mathbf{R})\right| \\
& =\sum_{i j}\left\langle\psi_{j}(\mathbf{r}, \mathbf{R})\right| \nabla_{\mathbf{R}}\left(\nabla_{\mathbf{R}}\left|\psi_{i}(\mathbf{r}, \mathbf{R})\right\rangle\right)\left|\psi_{i}(\mathbf{r}, \mathbf{R})\right\rangle\left\langle\psi_{j}(\mathbf{r}, \mathbf{R})\right| \\
& =\sum_{i j k}\left\langle\psi_{j}(\mathbf{r}, \mathbf{R})\right| \nabla_{\mathbf{R}}\left(\left|\psi_{k}(\mathbf{r}, \mathbf{R})\right\rangle\left\langle\psi_{k}(\mathbf{r}, \mathbf{R})\left|\nabla_{\mathbf{R}}\right| \psi_{i}(\mathbf{r}, \mathbf{R})\right\rangle\right)\left|\psi_{i}(\mathbf{r}, \mathbf{R})\right\rangle\left\langle\psi_{j}(\mathbf{r}, \mathbf{R})\right|
\end{aligned}
$$

where we have used the identity in the electronic subspace $\hat{\mathbb{1}}_{\mathrm{e}}=\sum_{k}\left|\psi_{k}(\mathbf{r}, \mathbf{R})\right\rangle\left\langle\psi_{k}(\mathbf{r}, \mathbf{R})\right|$. 
Noticing that in the last line of the above equation, $\nabla_{\mathbf{R}}$ will act on both terms inside $\left(\left|\psi_{k}(\mathbf{r}, \mathbf{R})\right\rangle\left\langle\psi_{k}(\mathbf{r}, \mathbf{R})\left|\nabla_{\mathbf{R}}\right| \psi_{i}(\mathbf{r}, \mathbf{R})\right\rangle=\left(\left|\psi_{k}(\mathbf{r}, \mathbf{R})\right\rangle \mathbf{d}_{k i}(\mathbf{R})\right)\right.$ that dependent on $\mathbf{R}$, leading to

$$
\begin{aligned}
\mathbf{D} & =\mathbf{d} \cdot \mathbf{d}+\left(\nabla_{\mathbf{R}} \cdot \mathbf{d}\right) \\
& =\mathbf{d} \cdot \mathbf{d}+\left(\nabla_{\mathbf{R}} \cdot \mathbf{d}\right)+\mathbf{d} \cdot \nabla_{\mathbf{R}}-\mathbf{d} \cdot \nabla_{\mathbf{R}} \\
& =\mathbf{d} \cdot \mathbf{d}+\nabla_{\mathbf{R}} \cdot \mathbf{d}-\mathbf{d} \cdot \nabla_{\mathbf{R}},
\end{aligned}
$$

where we used $\nabla_{\mathbf{R}} \cdot \mathbf{d}(\mathbf{R})|\varphi(\mathbf{R})\rangle=\left(\nabla_{\mathbf{R}} \cdot \mathbf{d}\right)|\varphi(\mathbf{R})\rangle+\mathbf{d} \cdot \nabla_{\mathbf{R}}|\varphi(\mathbf{R})\rangle$, hence, $\nabla_{\mathbf{R}} \cdot \mathbf{d}(\mathbf{R})=$ $\left(\nabla_{\mathbf{R}} \cdot \mathbf{d}\right)+\mathbf{d} \cdot \nabla_{\mathbf{R}}$. Using this in Eqn. S6, we obtain

$$
\begin{aligned}
\hat{\mathbf{T}}_{\mathbf{R}} & =-\frac{\hbar^{2}}{2 M}\left(\nabla_{\mathbf{R}}^{2} \otimes \hat{\mathbb{1}}_{\mathrm{e}}+\mathbf{d} \cdot \nabla_{\mathbf{R}}+\nabla_{\mathbf{R}} \cdot \mathbf{d}+\mathbf{d} \cdot \mathbf{d}\right) \\
& =-\frac{\hbar^{2}}{2 M}\left(\nabla_{\mathbf{R}} \otimes \hat{\mathbb{1}}_{\mathrm{e}}+\mathbf{d}\right)^{2}=\frac{1}{2 M}\left(-i \hbar \nabla_{\mathbf{R}} \otimes \hat{\mathbb{1}}_{\mathrm{e}}-i \hbar \mathbf{d}\right)^{2} .
\end{aligned}
$$

The molecular Hamiltonian can be written as

$$
\hat{H}_{\mathrm{M}}=\frac{1}{2 M}\left(\hat{\mathbf{P}} \otimes \hat{\mathbb{1}}_{\mathrm{e}}-i \hbar \mathbf{d}\right)^{2}+\sum_{i} E_{i}\left|\psi_{i}(\mathbf{r}, \mathbf{R})\right\rangle\left\langle\psi_{i}(\mathbf{r}, \mathbf{R})\right|
$$

where $\hat{\mathbf{P}}=-i \hbar \nabla_{\mathbf{R}}$. This equivalence was first shown in Ref. 1, and we use it explicitly in the main text (Eqn. 4).

\section{Non-adiabatic coupling between polarized Fock states}

The non-adiabatic couplings among the PFS (from Eqn. 9 of the main text) is $\left\langle n_{\alpha}\left|\nabla_{\mathbf{R}}\right| m_{\beta}\right\rangle$. Using the fact that the polarized Fock state $\left|n_{\alpha}\right\rangle$ is the displaced vacuum's Fock state $|n\rangle$,

with a displacement of $-q_{\alpha}^{0}=-\sqrt{\frac{2}{\hbar \omega_{\mathrm{c}}^{3}}} \chi \cdot \boldsymbol{\mu}_{\alpha}(\mathbf{R})$, such that $\left|n_{\alpha}\right\rangle=e^{-i\left(-q_{\alpha}^{0}\right) \hat{p} / \hbar}|n\rangle=e^{i q_{\alpha}^{0} \hat{p} / \hbar}|n\rangle$, 
we can evaluate the $\left\langle n_{\alpha}\left|\nabla_{\mathbf{R}}\right| m_{\beta}\right\rangle$ term as follows

$$
\begin{aligned}
\left\langle m_{\alpha}\left|\nabla_{\mathbf{R}}\right| n_{\alpha}\right\rangle & =\left\langle m_{\alpha}\left|\nabla_{\mathbf{R}} e^{i q_{\alpha}^{0} \hat{p} / \hbar}\right| n\right\rangle \\
& =\left\langle m_{\alpha}\left|\frac{i}{\hbar}\left(\nabla_{\mathbf{R}} q_{\alpha}^{0}\right) \hat{p} e^{i q_{\alpha}^{0} \hat{p} / \hbar}\right| n\right\rangle=\left\langle m_{\alpha}\left|\frac{i}{\hbar}\left(\nabla_{\mathbf{R}} q_{\alpha}^{0}\right) \hat{p}\right| n_{\alpha}\right\rangle \\
& =-\frac{1}{\hbar \omega_{\mathrm{c}}} \boldsymbol{\chi} \cdot \nabla_{\mathbf{R}} \boldsymbol{\mu}_{\alpha}(\mathbf{R})\left\langle m_{\alpha}\left|\hat{a}^{\dagger}-\hat{a}\right| n_{\alpha}\right\rangle \\
& =-\frac{1}{\hbar \omega_{\mathrm{c}}} \boldsymbol{\chi} \cdot \nabla_{\mathbf{R}} \boldsymbol{\mu}_{\alpha}(\mathbf{R})\left\langle m_{\alpha}\left|\hat{b}_{\alpha}^{\dagger}-\hat{b}_{\alpha}\right| n_{\alpha}\right\rangle .
\end{aligned}
$$

Note that $\nabla_{\mathbf{R}}$ only acts on $\mathbf{R}$ of $q_{\alpha}^{0}$, not on $\hat{p}$ because it belongs to the photonic DOF. To derive the above equation, we have used the fact that $\hat{p}=i \sqrt{\hbar \omega_{\mathrm{c}} / 2}\left(\hat{a}^{\dagger}-\hat{a}\right)$. Further, $\hat{b}_{\alpha}^{\dagger}=$

$\left(\sqrt{\frac{\omega_{c}}{\hbar}} \hat{q}_{\alpha}^{\prime}-i \sqrt{\frac{1}{\hbar \omega_{c}}} \hat{p}\right) / \sqrt{2}$ and $\hat{b}_{\alpha}=\left(\sqrt{\frac{\omega_{c}}{\hbar}} \hat{q}_{\alpha}^{\prime}+i \sqrt{\frac{1}{\hbar \omega_{c}}} \hat{p}\right) / \sqrt{2}$ are the creation and annihilation operators of the PFS $\left|n_{\alpha}\right\rangle$, with the photon field momentum operator $\hat{p}$ and polarized photon field coordinate operator $\hat{q}_{\alpha}^{\prime}=\hat{q}+q_{\alpha}^{0}(\mathbf{R})$ as we introduced in the main text. Thus, $\hat{a}^{\dagger}-\hat{a}=$ $\hat{b}_{\alpha}^{\dagger}-\hat{b}_{\alpha}$, which we have explicitly used in the above derivation. More specifically, the matrix elements of the non-adiabatic couplings in Eqn. S10 are

$$
\left\langle m_{\alpha}\left|\nabla_{\mathbf{R}}\right| n_{\alpha}\right\rangle=-\frac{1}{\hbar \omega_{\mathrm{c}}} \chi \cdot \nabla_{\mathbf{R}} \boldsymbol{\mu}_{\alpha}(\mathbf{R})\left[\sqrt{n_{\alpha}+1} \delta_{m_{\alpha}, n_{\alpha}+1}-\sqrt{n_{\alpha}} \delta_{m_{\alpha}, n_{\alpha}-1}\right]
$$

\section{Connection to The P A Hamiltonian}

The PF Hamiltonian under the $\left|\alpha, n_{\alpha}\right\rangle$ representation can be shown to adapt a P - A form Hamiltonian, similar to the polarized vacuum states discussed in Ref. 2. To simplify our task, here we focus on the two-level molecules in the main text, with the following Hamiltonian

$$
\begin{aligned}
\hat{H} & =\frac{1}{2 M}\left(\hat{\mathbf{P}}-i \hbar \sum_{\alpha, n_{\alpha}, m_{\alpha}} \mathbf{d}_{m_{\alpha} n_{\alpha}}\left|\alpha, m_{\alpha}\right\rangle\left\langle\alpha, n_{\alpha}\right|\right)^{2}+\sum_{\alpha, n_{\alpha}}\left(V_{\alpha}(\mathbf{R})+\left(n_{\alpha}+1 / 2\right) \hbar \omega_{\mathrm{c}}\right)\left|\alpha, n_{\alpha}\right\rangle\left\langle\alpha, n_{\alpha}\right| \\
& +\sum_{n_{\mathrm{I}}, m_{\mathrm{C}}}\left\langle m_{\mathrm{C}} \mid n_{\mathrm{I}}\right\rangle V_{\mathrm{IC}}(\mathbf{R})\left(\left|\mathrm{I}, n_{\mathrm{I}}\right\rangle\left\langle\mathrm{C}, m_{\mathrm{C}}|+| \mathrm{C}, m_{\mathrm{C}}\right\rangle\left\langle\mathrm{I}, n_{\mathrm{I}}\right|\right),
\end{aligned}
$$

where $\mathbf{d}_{m_{\alpha} n_{\alpha}}=\left\langle m_{\alpha}\left|\nabla_{\mathbf{R}}\right| n_{\alpha}\right\rangle$. 
To show that $\hat{H}$ has the "p.A" form, we perform an unitary transformation on $\hat{H}$ using the operator

$$
\hat{U}_{\theta}=\exp \left[-i \frac{\pi}{2} \sum_{\alpha} \hat{b}_{\alpha}^{\dagger} \hat{b}_{\alpha}|\alpha\rangle\langle\alpha|\right]
$$

where $\hat{b}_{\alpha}^{\dagger}$ and $\hat{b}_{\alpha}$ are the creation and annihilation operators of the PFS, respectively. Note that only $\left\langle\alpha, n_{\alpha}\right|$ and $\left|\alpha, n_{\alpha}\right\rangle$ are transformed by $\hat{U}_{\theta}$. Applying this transformation on $\left|\alpha, m_{\alpha}\right\rangle\left\langle\alpha, n_{\alpha}\right|$, we have

$$
\begin{aligned}
& \hat{U}_{\theta}^{\dagger}\left|\alpha, m_{\alpha}\right\rangle\left\langle\alpha, n_{\alpha}\left|\hat{U}_{\theta}=e^{i \frac{\pi}{2}\left(m_{\alpha}-n_{\alpha}\right)}\right| \alpha, m_{\alpha}\right\rangle\left\langle\alpha, n_{\alpha}\right| \\
& \hat{U}_{\theta}^{\dagger}\left|\mathrm{C}, m_{\mathrm{C}}\right\rangle\left\langle\mathrm{I}, n_{\mathrm{I}}\left|\hat{U}_{\theta}=e^{i \frac{\pi}{2}\left(m_{\mathrm{C}}-n_{\mathrm{I}}\right)}\right| \mathrm{C}, m_{\mathrm{C}}\right\rangle\left\langle\mathrm{I}, n_{\mathrm{I}}\right| .
\end{aligned}
$$

Using this transformation, the kinetic energy operator becomes

$$
\hat{T}_{\mathbf{R}}^{\prime}=\hat{U}_{\theta}^{\dagger} \hat{T}_{\mathbf{R}} \hat{U}_{\theta}=\hat{U}_{\theta}^{\dagger}(\hat{\mathbf{P}}-i \hbar \mathbf{d}) \hat{U}_{\theta} \hat{U}_{\theta}^{\dagger}(\hat{\mathbf{P}}-i \hbar \mathbf{d}) \hat{U}_{\theta}
$$

where $\hat{U}_{\theta}^{\dagger}(\hat{\mathbf{P}}-i \hbar \hat{\mathbf{d}}) \hat{U}_{\theta}$ is given as

$$
\begin{aligned}
\hat{U}_{\theta}^{\dagger}(\hat{\mathbf{P}}-i \hbar \hat{d}) \hat{U}_{\theta} & =\hat{\mathbf{P}}-i \hbar \sum_{\alpha, n_{\alpha}, m_{\alpha}} \mathbf{d}_{m_{\alpha} n_{\alpha}} \hat{U}_{\theta}^{\dagger}\left|\alpha, m_{\alpha}\right\rangle\left\langle\alpha, n_{\alpha}\right| \hat{U}_{\theta} \\
& =\hat{\mathbf{P}}-i \hbar \sum_{\alpha, n_{\alpha}, m_{\alpha}} e^{i \frac{\pi}{2}\left(m_{\alpha}-n_{\alpha}\right)} \mathbf{d}_{m_{\alpha} n_{\alpha}}\left|\alpha, m_{\alpha}\right\rangle\left\langle\alpha, n_{\alpha}\right| .
\end{aligned}
$$

In addition, using Eqn. S10 we can show that

$$
\begin{aligned}
i \hbar e^{i \frac{\pi}{2}\left(m_{\alpha}-n_{\alpha}\right)} \mathbf{d}_{m_{\alpha} n_{\alpha}} & =i \hbar e^{i \frac{\pi}{2}\left(m_{\alpha}-n_{\alpha}\right)}\left\langle m_{\alpha}\left|\nabla_{\mathbf{R}}\right| n_{\alpha}\right\rangle \\
& =-\frac{i \hbar}{\hbar \omega_{\mathrm{c}}} e^{i \frac{\pi}{2}\left(m_{\alpha}-n_{\alpha}\right)} \boldsymbol{\chi} \cdot \nabla_{\mathbf{R}} \boldsymbol{\mu}_{\alpha}(\mathbf{R})\left[\sqrt{n_{\alpha}+1} \delta_{m_{\alpha}, n_{\alpha}+1}-\sqrt{n_{\alpha}} \delta_{m_{\alpha}, n_{\alpha}-1}\right] \\
& =-\frac{i}{\omega_{\mathrm{c}}} \boldsymbol{\chi} \cdot \nabla_{\mathbf{R}} \boldsymbol{\mu}_{\alpha}(\mathbf{R})\left[e^{i \frac{\pi}{2}} \sqrt{n_{\alpha}+1} \delta_{m_{\alpha}, n_{\alpha}+1}-e^{-i \frac{\pi}{2}} \sqrt{n_{\alpha}} \delta_{m_{\alpha}, n_{\alpha}-1}\right] \\
& =\frac{1}{\omega_{\mathrm{c}}} \boldsymbol{\chi} \cdot \nabla_{\mathbf{R}} \boldsymbol{\mu}_{\alpha}(\mathbf{R})\left\langle m_{\alpha}\left|\hat{b}_{\alpha}^{\dagger}+\hat{b}_{\alpha}\right| n_{\alpha}\right\rangle=\nabla_{\mathbf{R}} \boldsymbol{\mu}_{\alpha}(\mathbf{R})\left\langle m_{\alpha}\left|\mathbf{A}_{\alpha}\right| n_{\alpha}\right\rangle
\end{aligned}
$$


where the vector potential of the polarized field is

$$
\mathbf{A}_{\alpha}=\frac{\chi}{\omega_{\mathrm{c}}}\left(\hat{b}_{\alpha}^{\dagger}+\hat{b}_{\alpha}\right)
$$

in analogous of the quantized transverse vector potential under the the long wavelength limit (see Eqn. S58 and S60). Overall, the kinetic energy operator becomes

$$
\begin{aligned}
\hat{T}_{R}^{\prime} & =\frac{1}{2 M}\left(\hat{\mathbf{P}}-\sum_{\alpha, n_{\alpha}, m_{\alpha}} \nabla_{\mathbf{R}} \boldsymbol{\mu}_{\alpha}(\mathbf{R})\left\langle m_{\alpha}\left|\mathbf{A}_{\alpha}\right| n_{\alpha}\right\rangle\left|\alpha, m_{\alpha}\right\rangle\left\langle\alpha, n_{\alpha}\right|\right)^{2} \\
& =\frac{1}{2 M}\left(\hat{\mathbf{P}}-\sum_{\alpha} \nabla_{\mathbf{R}} \boldsymbol{\mu}_{\alpha}(\mathbf{R}) \mathbf{A}_{\alpha}|\alpha\rangle\langle\alpha|\right)^{2}
\end{aligned}
$$

Hence, through $U_{\theta}$, we restore the nuclear kinetic energy operator in the " $\mathrm{P} \cdot \mathrm{A}$ " form. This is of course, different than the usual "P · A" Hamiltonian defined in Eqn. S62, where the momentum of both electron and nuclear (all charged particles in the matter) couples to the vector potential through the charges of these particles (i.e., a minimal coupling structure). Here, $\hat{T}_{\mathbf{R}}^{\prime}$ shows that the nuclear momentum is coupled to the vector potential of the polarized field (defined by the operator $\hat{b}^{\dagger}$ and $\hat{b}$ ) through the negative gradient of dipole. A more fundamental reason for the difference between these two types of "P $A$ " coupling is that Eqn. S62 is written down in a complete basis, and Eqn. S19 is written in the truncated electronic Hilbert subspace. ${ }^{3}$

Further, we perform the transformation for the polariton Hamiltonian $H_{\mathrm{pl}}=\hat{H}-\hat{T}_{\mathbf{R}}$ as follows

$$
\begin{aligned}
& \hat{H}_{\mathrm{pl}}^{\prime}=\hat{U}_{\theta}^{\dagger} \hat{H}_{\mathrm{pl}} \hat{U}_{\theta} \\
& =\sum_{\alpha, n_{\alpha}}\left(V_{\alpha}(\mathbf{R})+\left(n_{\alpha}+1 / 2\right) \hbar \omega_{\mathrm{c}}\right)\left|\alpha, n_{\alpha}\right\rangle\left\langle\alpha, n_{\alpha}\right|+\sum_{n_{\mathrm{I}}, m_{\mathrm{C}}} V_{\mathrm{IC}}(\mathbf{R})\left\langle m_{\mathrm{C}} \mid n_{\mathrm{I}}\right\rangle e^{i \frac{\pi}{2}\left(m_{\mathrm{C}}-n_{\mathrm{I}}\right)}\left(\left|\mathrm{I}, n_{\mathrm{I}}\right\rangle\left\langle\mathrm{C}, m_{\mathrm{C}}\right|+\text { h.c }\right) \\
& =\sum_{\alpha, n_{\alpha}}\left(V_{\alpha}(\mathbf{R})+\left(n_{\alpha}+1 / 2\right) \hbar \omega_{\mathrm{c}}\right)\left|\alpha, n_{\alpha}\right\rangle\left\langle\alpha, n_{\alpha}\right|+\sum_{n_{\mathrm{I}}, m_{\mathrm{C}}} V_{\mathrm{IC}}(\mathbf{R})\left\langle m_{\mathrm{C}} \mid n_{\mathrm{I}}\right\rangle i^{\left(m_{\mathrm{C}}-n_{\mathrm{I}}\right)}\left(\left|\mathrm{I}, n_{\mathrm{I}}\right\rangle\left\langle\mathrm{C}, m_{\mathrm{C}}\right|+\text { h.c }\right),
\end{aligned}
$$

where "h.c" stands for the hermitian conjugate of $\left|\mathrm{I}, n_{\mathrm{I}}\right\rangle\left\langle\mathrm{C}, m_{\mathrm{C}}\right|$, which is $\left|\mathrm{C}, m_{\mathrm{C}}\right\rangle\left\langle\mathrm{I}, n_{\mathrm{I}}\right|$, and 
the fact that both $m_{\mathrm{I}}$ and $n_{\mathrm{C}}$ are integer help to reduce the phase $e^{i \frac{\pi}{2}\left(m_{\mathrm{C}}-n_{\mathrm{I}}\right)}$ to $i^{\left(m_{\mathrm{C}}-n_{\mathrm{I}}\right)}$. This tells us that $\hat{H}_{\mathrm{pl}}^{\prime}$ differs from $\hat{H}_{\mathrm{pl}}$ by a complex phase for all the off-diagonal electronic coupling.

\section{Connection to the Polaron Transformation}

The PF Hamiltonian in the diabatic basis $\{|\alpha\rangle,|\beta\rangle\}$ considered in the main text is

$$
\begin{aligned}
\hat{H} & =\hat{T}_{\mathbf{R}}+\sum_{\alpha} V_{\alpha \alpha}(\mathbf{R})|\alpha\rangle\left\langle\alpha\left|+\sum_{\alpha \neq \beta} V_{\alpha \beta}(\mathbf{R})\right| \alpha\right\rangle\langle\beta| \\
& +\frac{\hat{p}^{2}}{2}+\sum_{\alpha}\left[\frac{\omega_{\mathrm{c}}^{2}}{2}\left(\hat{q}+\sqrt{\frac{2}{\hbar \omega_{\mathrm{c}}^{3}}} \boldsymbol{\chi} \cdot \boldsymbol{\mu}_{\alpha}(\mathbf{R})\right)^{2}\right]|\alpha\rangle\langle\alpha| .
\end{aligned}
$$

We consider the following polaron-like transformation (similar to Ref. 4) with the unitary operator

$$
\hat{U}_{\text {pol }}=\exp \left[\frac{i}{\hbar}\left(\sum_{\alpha} \sqrt{\frac{2}{\hbar \omega_{\mathrm{c}}^{3}}} \boldsymbol{\chi} \cdot \boldsymbol{\mu}_{\alpha}(\mathbf{R})|\alpha\rangle\langle\alpha|\right) \hat{p}\right]=\exp \left[\frac{i}{\hbar} \sum_{\alpha} q_{\alpha}^{0}(\mathbf{R})|\alpha\rangle\langle\alpha| \hat{p}\right]
$$

where $q_{\alpha}^{0}(\mathbf{R})=\sum_{\alpha} \sqrt{\frac{2}{\hbar \omega_{\mathrm{c}}^{3}}} \chi \cdot \boldsymbol{\mu}_{\alpha}(\mathbf{R})$ is the photonic displacement associated with state $|\alpha\rangle$. The polarized Fock states defined in the main text is

$$
\hat{U}_{\mathrm{pol}}|\alpha\rangle|n\rangle=|\alpha\rangle\left|n_{\alpha}(\mathbf{R})\right\rangle, \quad\left\langle m_{\beta}(\mathbf{R})\right|\langle\beta|=\langle m|\langle\beta| \hat{U}_{\text {pol }}^{\dagger}
$$

Using the polarized Fock state to evaluate the Hamiltonian, we have

$$
\hat{H}=\sum_{\alpha, \beta} \sum_{n_{\alpha}, m_{\beta}}|\alpha\rangle\left|n_{\alpha}\right\rangle H_{\alpha, \beta, n_{\alpha}, m_{\beta}}\left\langle m_{\beta}\right|\langle\beta|,
$$

where the matrix elements are

$$
H_{\alpha, \beta, n_{\alpha}, m_{\beta}}=\left\langle\alpha\left|\left\langle n_{\alpha}|\hat{H}| m_{\beta}\right\rangle\right| \beta\right\rangle=\left\langle\alpha\left|\left\langle n\left|\hat{U}_{\text {pol }}^{\dagger} \hat{H} \hat{U}_{\text {pol }}\right| m\right\rangle\right| \beta\right\rangle
$$


Thus, the matrix element of $\hat{H}$ in the $\left|\alpha, n_{\alpha}\right\rangle$ is the same as the matrix element of $\hat{H}^{\prime}=\hat{U}_{\mathrm{pol}}^{\dagger} \hat{H} \hat{U}_{\mathrm{pol}}$ in the $|\alpha, n\rangle$ representation. This is the connection of PFS and the polaron transformed Hamiltonian $\hat{H}^{\prime}$. Below, we derive the detailed expression of $\hat{H}^{\prime}$. Using the Baker-Hausdorff identity

$$
e^{\hat{A}} \hat{B} e^{-\hat{A}}=\hat{B}+[\hat{A}, \hat{B}]+\frac{1}{2 !}[\hat{A},[\hat{A}, \hat{B}]]+\ldots
$$

the nuclear momentum operator $\hat{P}$ is transformed as

$$
\hat{U}_{\mathrm{pol}}^{\dagger} \hat{\mathbf{P}} \hat{U}_{\mathrm{pol}}=\hat{\mathbf{P}}-\frac{i}{\hbar} \hat{p}\left[\sum_{\alpha} q_{\alpha}^{0}|\alpha\rangle\langle\alpha|, \hat{\mathbf{P}}\right]+\frac{1}{2 !}\left(\frac{i}{\hbar} \hat{p}\right)^{2}\left[\sum_{\alpha} q_{\alpha}^{0}|\alpha\rangle\langle\alpha|,\left[\sum_{\alpha} q_{\alpha}^{0}|\alpha\rangle\langle\alpha|, \hat{P}\right]\right]+\ldots
$$

With $\left[\sum_{\alpha} q_{\alpha}^{0}(\mathbf{R})|\alpha\rangle\langle\alpha|, \hat{\mathbf{P}}\right]=i \hbar \sum_{\alpha} \nabla_{\mathbf{R}} q_{\alpha}^{0}(\mathbf{R})|\alpha\rangle\langle\alpha|$ and $\left[\sum_{\alpha} q_{\alpha}^{0}(\mathbf{R})|\alpha\rangle\left\langle\alpha\left|, i \hbar \sum_{\alpha} \nabla_{\mathbf{R}} q_{\alpha}^{0}(\mathbf{R})\right| \alpha\right\rangle\langle\alpha|\right]=$ 0, only the first two terms in Eqn. S27 are non-zero, leading to

$$
\hat{U}_{\mathrm{pol}}^{\dagger} \hat{\mathbf{P}} \hat{U}_{\mathrm{pol}}=\hat{\mathbf{P}}+\hat{p} \sum_{\alpha} \nabla_{\mathbf{R}} q_{\alpha}^{0}(\mathbf{R})|\alpha\rangle\left\langle\alpha\left|=\hat{\mathbf{P}}+i \sum_{\alpha} \frac{\boldsymbol{\chi}}{\omega_{\mathrm{c}}} \cdot \nabla_{\mathbf{R}} \boldsymbol{\mu}_{\alpha}(\mathbf{R})\left(\hat{a}^{\dagger}-\hat{a}\right)\right| \alpha\right\rangle\langle\alpha|
$$

Hence, $\hat{U}_{\text {pol }}$ is a momentum boost operator for the nuclear DOF, because it explicitly contains $q_{\alpha}^{0}(\mathbf{R})$. Meanwhile, it is also a displacement operator for the photonic DOF, because it contains photonic momentum operator $\hat{p}$, which shifts the photonic coordinate by $-q_{\alpha}^{0}$.

Using the above result of $\hat{U}_{\mathrm{pol}}^{\dagger} \hat{\mathbf{P}} \hat{U}_{\mathrm{pol}}$ and the fact that $\hat{U}_{\mathrm{pol}}^{\dagger} \hat{U}_{\mathrm{pol}}=1$, the polaron-transformed nuclear kinetic energy operator is

$$
\begin{aligned}
& \hat{U}_{\mathrm{pol}}^{\dagger} \hat{T}_{\mathbf{R}} \hat{U}_{\mathrm{pol}}=\frac{1}{2 M} \hat{U}_{\mathrm{pol}}^{\dagger} \hat{\mathbf{P}} \hat{U}_{\mathrm{pol}} \hat{U}_{\mathrm{pol}}^{\dagger} \hat{\mathbf{P}} \hat{U}_{\mathrm{pol}} \\
& =\frac{1}{2 M}\left(\hat{\mathbf{P}}+i \sum_{\alpha} \frac{\boldsymbol{\chi}}{\omega_{\mathrm{c}}} \cdot \nabla_{R} \boldsymbol{\mu}_{\alpha}(\mathbf{R})\left(\hat{a}^{\dagger}-\hat{a}\right)|\alpha\rangle\langle\alpha|\right)^{2} .
\end{aligned}
$$


The polaron transformation on the operator $|\alpha\rangle\langle\beta|$ is

$$
\hat{U}_{\mathrm{pol}}^{\dagger}|\alpha\rangle\left\langle\beta\left|\hat{U}_{\mathrm{pol}}=e^{\frac{i}{\hbar} \hat{p} \sqrt{\frac{2}{\hbar \omega_{\mathrm{c}}^{3}}} \chi \cdot\left(\mu_{\alpha}(\mathbf{R})-\mu_{\beta}(\mathbf{R})\right)}\right| \alpha\right\rangle\langle\beta|
$$

Hence, the diagonal diabatic potential operator transforms as

$$
\hat{U}_{\mathrm{pol}}^{\dagger}\left(\sum_{\alpha} V_{\alpha \alpha}(\mathbf{R})|\alpha\rangle\langle\alpha|\right) \hat{U}_{\mathrm{pol}}=\sum_{\alpha} V_{\alpha \alpha}(\mathbf{R})|\alpha\rangle\langle\alpha|,
$$

and the off-diagonal diabatic potential is transformed as

$$
\hat{U}_{\text {pol }}^{\dagger}\left(\sum_{\alpha \neq \beta} V_{\alpha \beta}(\mathbf{R})|\alpha\rangle\langle\beta|\right) \hat{U}_{\text {pol }}=V_{\alpha \beta}(\mathbf{R})\left(e^{\frac{i}{\hbar} \hat{p} \sqrt{\frac{2}{\hbar \omega_{c}^{3}}} x \cdot\left(\mu_{\alpha}(R)-\mu_{\beta}(\mathbf{R})\right)}|\alpha\rangle\langle\beta|+\text { h.c }\right),
$$

where "h.c" stands for the hermitian conjugate of the previous term.

Finally, noting that the $\hat{U}_{\text {pol }}^{\dagger}$ is a translation operator for the photon field with a displacement of $-q_{\alpha}^{0}(\mathbf{R})$ for state $|\alpha\rangle$, we well as Eqn. S30, we obtain

$$
\hat{U}_{\mathrm{pol}}^{\dagger}\left[\frac{\hat{p}^{2}}{2}+\sum_{\alpha} \frac{\omega_{\mathrm{c}}^{2}}{2}\left(\hat{q}+q_{\alpha}^{0}(\mathbf{R})\right)^{2}|\alpha\rangle\langle\alpha|\right] \hat{U}_{\mathrm{pol}}=\frac{\hat{p}^{2}}{2}+\frac{1}{2} \omega_{\mathrm{c}}^{2} \hat{q}^{2}=\left(\hat{a}^{\dagger} \hat{a}+\frac{1}{2}\right) \hbar \omega_{\mathrm{c}}
$$

Using Eqn. S29-S33, we obtain the polaron transformed PF Hamiltonian $\hat{H}^{\prime}$. Using the $|\alpha, n\rangle$ basis to evaluate $\hat{H}^{\prime}$ results in the same matrix elements as using $\left|\alpha, n_{\alpha}\right\rangle$ to evaluate $\hat{H}$.

\section{Analytical Expressions of the two-state system}

For the two state molecular system considered here, the $\hat{\boldsymbol{\mu}}$ in the adiabatic representation $\{|g\rangle,|e\rangle\}$ is

$$
\hat{\boldsymbol{\mu}}=\left[\begin{array}{ll}
\boldsymbol{\mu}_{g g} & \boldsymbol{\mu}_{g e} \\
\boldsymbol{\mu}_{e g} & \boldsymbol{\mu}_{e e}
\end{array}\right]
$$


The unitary matrix $\hat{U}_{\mu}$ that diagonalizes the above $2 \times 2$ adiabatic dipole moment is given by

$$
\hat{U}_{\mu}=\left[\begin{array}{cc}
\cos \theta_{\mu} & \sin \theta_{\mu} \\
-\sin \theta_{\mu} & \cos \theta_{\mu}
\end{array}\right],
$$

where the mixing angle $\theta_{\mu}$ is given by

$$
\theta_{\mu}=\frac{1}{2} \tan ^{-1}\left[\frac{2 \boldsymbol{\mu}_{e g}}{\boldsymbol{\mu}_{e e}-\boldsymbol{\mu}_{g g}}\right]
$$

The MH diabatic states $\{|\mathrm{C}\rangle,|\mathrm{I}\rangle\}$ are the unitary transformation (rotation) of the adiabatic states $\{|g\rangle,|e\rangle\}$ through

$$
\begin{gathered}
|\mathrm{I}\rangle=\cos \theta_{\mu}|g\rangle+\sin \theta_{\mu}|e\rangle \\
|\mathrm{C}\rangle=-\sin \theta_{\mu}|g\rangle+\cos \theta_{\mu}|e\rangle .
\end{gathered}
$$

The unitary operator $\hat{U}_{\mu}$ diagonalizes the adiabatic dipole and gives

$$
\begin{aligned}
{\left[\begin{array}{cc}
\boldsymbol{\mu}_{\mathrm{I}} & 0 \\
0 & \boldsymbol{\mu}_{\mathrm{C}}
\end{array}\right] } & \equiv \hat{U}_{\mu}^{\dagger}\left[\begin{array}{cc}
\boldsymbol{\mu}_{g g} & \boldsymbol{\mu}_{g e} \\
\boldsymbol{\mu}_{e g} & \boldsymbol{\mu}_{e e}
\end{array}\right] \hat{U}_{\mu}=\left[\begin{array}{cc}
\overline{\boldsymbol{\mu}}-\frac{1}{2} \Delta \boldsymbol{\mu} & 0 \\
0 & \overline{\boldsymbol{\mu}}+\frac{1}{2} \Delta \boldsymbol{\mu}
\end{array}\right] \\
& =\overline{\boldsymbol{\mu}} \mathbb{1}-\frac{1}{2} \Delta \boldsymbol{\mu} \hat{\sigma}_{z},
\end{aligned}
$$

where $\mathbb{1}$ and $\hat{\sigma}_{z}$ are the $2 \times 2$ identity matrix and the Pauli z-matrix, respectively, and

$$
\begin{aligned}
\overline{\boldsymbol{\mu}} & \equiv \frac{1}{2}\left(\boldsymbol{\mu}_{g g}+\boldsymbol{\mu}_{e e}\right) \\
\Delta \boldsymbol{\mu} & \equiv \sqrt{\left[\boldsymbol{\mu}_{e e}-\boldsymbol{\mu}_{g g}\right]^{2}+4 \boldsymbol{\mu}_{e g}{ }^{2}}=\boldsymbol{\mu}_{\mathrm{I}}-\boldsymbol{\mu}_{\mathrm{C}} .
\end{aligned}
$$

In addition, the matrix elements of the electronic Hamiltonian $\hat{H}_{\mathrm{el}}=\hat{H}-\hat{T}_{\mathrm{R}}$ (see Eqn. 
2 in the main text) in the $\mathrm{MH}$ representation is given by

$$
\left[\begin{array}{cc}
V_{\mathrm{I}} & V_{\mathrm{IC}} \\
V_{\mathrm{CI}} & V_{\mathrm{C}}
\end{array}\right] \equiv \hat{U}_{\mu}^{\dagger}\left[\begin{array}{cc}
E_{g} & 0 \\
0 & E_{e}
\end{array}\right] \hat{U}_{\mu}=\left[\begin{array}{cc}
E_{g} \cos ^{2} \theta_{\mu}+E_{e} \sin ^{2} \theta_{\mu} & \Delta E \cos \theta_{\mu} \sin \theta_{\mu} \\
\Delta E \cos \theta_{\mu} \sin \theta_{\mu} & E_{e} \cos ^{2} \theta_{\mu}+E_{g} \sin ^{2} \theta_{\mu}
\end{array}\right]
$$

where $\Delta E \equiv E_{e}(R)-E_{g}(R)$. Using the mixing angle in Eqn. S36, the diabatic coupling $V_{\mathrm{IC}}=\Delta E \cos \theta_{\mu} \sin \theta_{\mu}$ is evaluated as

$$
V_{\mathrm{IC}}=\frac{\boldsymbol{\mu}_{e g}\left[E_{e}-E_{g}\right]}{\sqrt{\left[\boldsymbol{\mu}_{e}-\boldsymbol{\mu}_{g}\right]^{2}+4 \boldsymbol{\mu}_{e g}^{2}}}=\boldsymbol{\mu}_{e g} \frac{\Delta E}{\Delta \boldsymbol{\mu}},
$$

where $\boldsymbol{\Delta} \boldsymbol{\mu}$ is defined in Eqn. S39.

Under the special case of the two states molecule, the polaron transform operator in Eqn. S22 is

$$
\hat{U}_{\mathrm{pol}}=\exp \left[-\frac{i}{\hbar} \sqrt{\frac{2}{\hbar \omega_{\mathrm{c}}^{3}}} \boldsymbol{\chi} \cdot\left(\overline{\boldsymbol{\mu}} \mathbf{I}-\frac{1}{2} \Delta \boldsymbol{\mu} \hat{\sigma}_{z}\right) \hat{p}\right]
$$

Under the special case where $\overline{\boldsymbol{\mu}}=0$ (for example, the atomic system where $\boldsymbol{\mu}_{e e}=\boldsymbol{\mu}_{g g}=0$ ), the above operator reduces to the displacement operator used in the generalized RWA. ${ }^{5,6}$

\section{Computational Details}

The polariton potential energy surfaces are computed using the diabatic-Fock state representation $\{|\alpha\rangle \otimes|n\rangle\}$ (with $|\alpha\rangle \in\{|\mathrm{I}\rangle,|\mathrm{C}\rangle\}$ for LiF model molecular system) as well as using the polarized Fock-States representation $\left\{|\alpha\rangle \otimes\left|n_{\alpha}\right\rangle\right\}$ introduced in this work. We use the Fourier Grid Hamiltonian (FGH) approach ${ }^{7,8}$ to represent the total light-matter interaction Hamiltonian with grid basis $\left\{\left|R_{i}\right\rangle\right\}$ to represent the nuclear degrees of freedom. In this work, we have 4096 grid points spanning over the range of $R \in[1.8,40]$ a.u. to ensure that the results are not contaminated by any spurious reflection of the nuclear wavepacket. During the entire simulation, the wave packet never propagate to the region that is close to the boundary of the edge of the grid points, and the absorption boundary is not used in the 

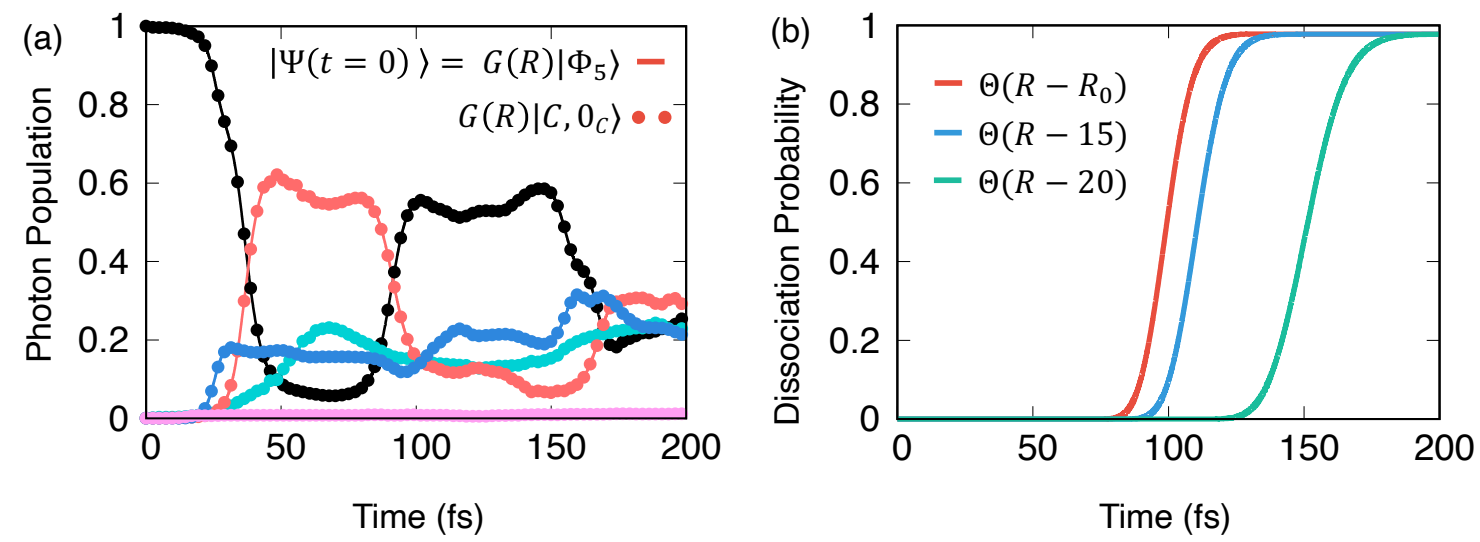

Figure S1: (a) Initial polariton wave-packet prepared as $G(R) \otimes\left|\Phi_{5}\right\rangle$ (solid lines) or $G(R) \otimes$ $\left|\mathrm{C}, 0_{\mathrm{C}}\right\rangle$ (dotted lines), both produce identical results because $\left|\Phi_{5}\right\rangle \approx\left|\mathrm{C}, 0_{\mathrm{C}}\right\rangle$ with nuclear configurations near the Franck-Condon region (when using $\hbar \omega_{\mathrm{c}}=1.5 \mathrm{eV}$ ). (b) Long-time dissociation probability remains unchanged when using the Heaviside function $\Theta(R)$ centered at $R_{0}, 15$ a.u. or 20 a.u. in when the light-matter coupling is $\chi=0.03$ a.u.

simulation. Further, we have used 20-100 vacuum Fock states when using the diabatic-Fock state representation while we only used 2-6 polarized Fock states when using the polarized Fock-States representation (which generate converged results that are visually identical to those obtained with a large amount of Fock states). The quantum dynamics of the moleculecavity hybrid system is propagated using the Split-Operator method ${ }^{8}$ with a nuclear time step of $d t=0.5$ a.u. to ensure converged results.

The initial condition of photoexcitation through out this work has been prepared by a vertical Franck-Condon excitation from the ground vibronic state, $\left|v_{0}\right\rangle \otimes\left|\Phi_{0}\right\rangle \approx G(R) \otimes$ $\left|\mathrm{I}, 0_{\mathrm{I}}\right\rangle$ where $\left|v_{0}\right\rangle$ is the ground vibronic state approximated by a Gaussian function $G(R)=$ $N_{\mathrm{R}} \exp \left(-\alpha\left(R-R_{g}\right)^{2}\right)$ with $R_{g}=3.01$ a.u. and $\alpha=19.12$ a.u. and $N_{\mathrm{R}}$ is a normalization constant. The parameters $\alpha$ and $R_{g}$ has been obtained by fitting the nuclear part of the ground-state wavefunction, which was obtained numerically exactly. For Fig. 2 in the maintext, the initial wavepacket is prepared as $G(R) \otimes\left|\Phi_{5}\right\rangle$ (when $\hbar \omega_{\mathrm{c}}=1.5 \mathrm{eV}$ ), while in Fig. 3 it is prepared as $G(R) \otimes\left|\Phi_{1}\right\rangle$ (when $\left.\hbar \omega_{\mathrm{c}}=7.5 \mathrm{eV}\right)$. Note that in either cases, the initial wavepacket can be equivalently expressed as $G(R) \otimes\left|\mathrm{C}, 0_{\mathrm{C}}\right\rangle$, which will provide visually 
indistinguishable results as shown in Fig. S1(b). This is because that near the FranckCondon region, $\left|\mathrm{C}, 0_{\mathrm{C}}\right\rangle \approx\left|\Phi_{5}\right\rangle$ when $\hbar \omega_{\mathrm{c}}=1.5 \mathrm{eV}$ and $\left|\mathrm{C}, 0_{\mathrm{C}}\right\rangle \approx\left|\Phi_{1}\right\rangle$ when $\hbar \omega_{\mathrm{c}}=7.5 \mathrm{eV}$, i.e., the polariton state is predominantly having the character of $\left|\mathrm{C}, 0_{\mathrm{C}}\right\rangle$.

For computing the dissociation probability (presented in Fig. 3b in the main text) defined as $P_{\text {dis }}(t)=\left\langle\Psi(t) \mid \Phi_{0}(R)\right\rangle\left\langle\Phi_{0}(R)\left|\Theta\left(R-R_{0}\right)\right| \Psi(t)\right\rangle$, where $\Theta$ is the Heaviside function chosen to be centered around $R_{0},\left|\Phi_{0}(R)\right\rangle$ is the polaritonic ground state for the molecule-cavity hybrid system, and $|\Psi(t)\rangle$ is the time dependent quantum state of the hybrid system. We note that using a Heaviside function centered around other nuclear position (rather than using $R_{0}$ ) does not affect the long time dissociation probability. This is demonstrated in Fig. S1(b) by choosing the Heaviside function centered at $R_{0} \sim 13.5$ a.u., 15 a.u., or 20 a.u. at $\chi=0.03$ a.u., all of which provides the same long-time dissociation probability.

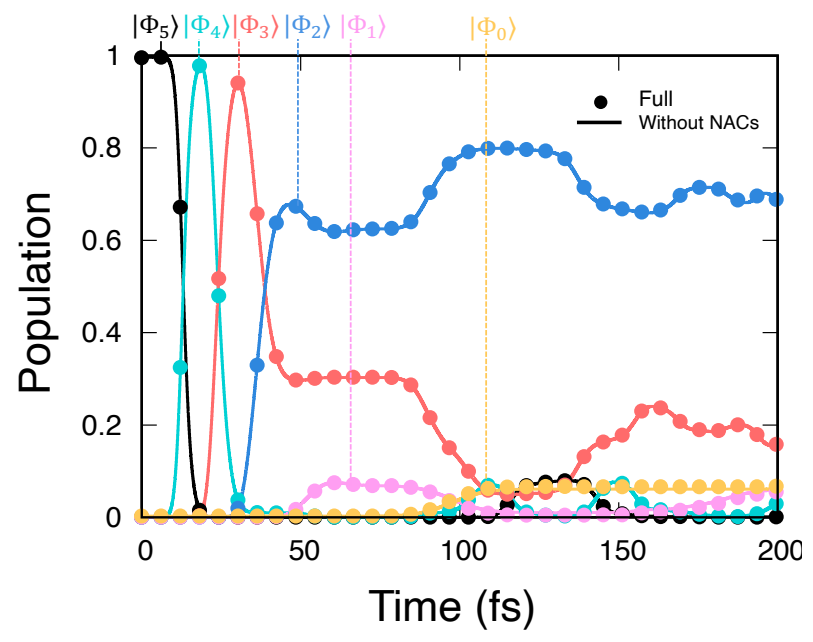

Figure S2: The impact of the non-adiabatic coupling on polariton quantum dynamics. All parameters are the same as those in Fig. 2 in the main text, with $\chi=0.004$ a.u. The timedependent population of polaritonic states computed using the full Hamiltonian in Eqn. S12 (solid lines) and the approximate Hamiltonian (dots) in Eqn. S43 that ignores non-adiabatic couplings between polarized Fock states.

\section{The Effect of Non-adiabatic couplings between polarized Fock states}

From the expression in Eqn. S50, one can clearly see that quantum states $\left\{\left|\alpha, n_{\alpha}\right\rangle\right\}$ are coupled through $\left\langle m_{\mathrm{C}} \mid n_{\mathrm{I}}\right\rangle V_{\mathrm{IC}}(\mathbf{R})$ in $\hat{H}_{\mathrm{pl}}$ and $\left\langle m_{\alpha}\left|\nabla_{\mathbf{R}}\right| n_{\alpha}\right\rangle$ in $\hat{T}_{\mathbf{R}}$. Here, we numerically demon- 
strate that the non-adiabatic coupling $\left\langle m_{\alpha}\left|\nabla_{\mathbf{R}}\right| n_{\alpha}\right\rangle$ between polarized Fock states given in Eqn. S10 does not play an important role in the light-matter quantum dynamics for the range of parameters used in this work. Their role, however, should not be overlooked and will be explored in future.

To demonstrate this point, we perform quantum dynamics simulations that ignores these non-adiabatic couplings through the following Hamiltonian for the LiF molecule coupled to the cavity,

$$
\begin{aligned}
& \hat{H} \approx \hat{T}_{R}+\sum_{\alpha, n_{\alpha}}\left(V_{\alpha}(R)+\left(n_{\alpha}+1 / 2\right) \hbar \omega_{\mathrm{c}}\right)\left|\alpha, n_{\alpha}\right\rangle\left\langle\alpha, n_{\alpha}\right| \\
& +\sum_{n_{\mathrm{I}}, m_{\mathrm{C}}}\left\langle m_{\mathrm{C}} \mid n_{\mathrm{I}}\right\rangle V_{\mathrm{IC}}(R)\left(\left|\mathrm{I}, n_{\mathrm{I}}\right\rangle\left\langle\mathrm{C}, m_{\mathrm{C}}|+| \mathrm{C}, m_{\mathrm{C}}\right\rangle\left\langle\mathrm{I}, n_{\mathrm{I}}\right|\right) .
\end{aligned}
$$

Fig. S2 presents the time-dependent population of polaritonic states $\left|\Phi_{j}\right\rangle$ at $\hbar \omega_{\mathrm{c}}=1.5$ $\mathrm{eV}$ and $\chi=0.004$ a.u. computed from the full Hamiltonian in Eqn. S12 and with the approximated Hamiltonian in Eqn. S43. Both Hamiltonians provide visually identical results, indicating that these non-adiabatic couplings are less important for the model system in this particular parameter regime. We have also carefully checked all of the results presented in the main text and find the same conclusion.

\section{The Energy splitting at the molecular avoided crossing}

Due to the state-dependent polarization of the photon field, the energy splitting at the molecular avoided crossing $\left(R_{0}\right)$ is modified by increasing the light-matter coupling $\chi$, as we explored in Fig. 3 of the main text.

Fig. S3 presents the numerical results (dots) of energy splitting $\Delta \mathcal{E}$ between $\left|\Phi_{0}\right\rangle$ and $\left|\Phi_{1}\right\rangle$ at $R=R_{0} \approx 13.5$ a.u. as a function of $\chi$. We compare this numerical results with the analytical expression (solid line) of $2 V_{\mathrm{IC}}\left(R_{0}\right)\left\langle 0_{\mathrm{I}} \mid 0_{\mathrm{C}}\right\rangle$, where $\Delta \mathcal{E}\left(R_{0}\right)=2 V_{\mathrm{IC}}\left(R_{0}\right)$ is the splitting for the bare molecular system (because at $R_{0}$, the diabatic states become degenerate $\left.V_{\mathrm{I}}\left(R_{0}\right)=V_{\mathrm{C}}\left(R_{0}\right)\right)$, and $\left\langle 0_{\mathrm{I}} \mid 0_{\mathrm{C}}\right\rangle$ describes the effects from the light-matter interaction 


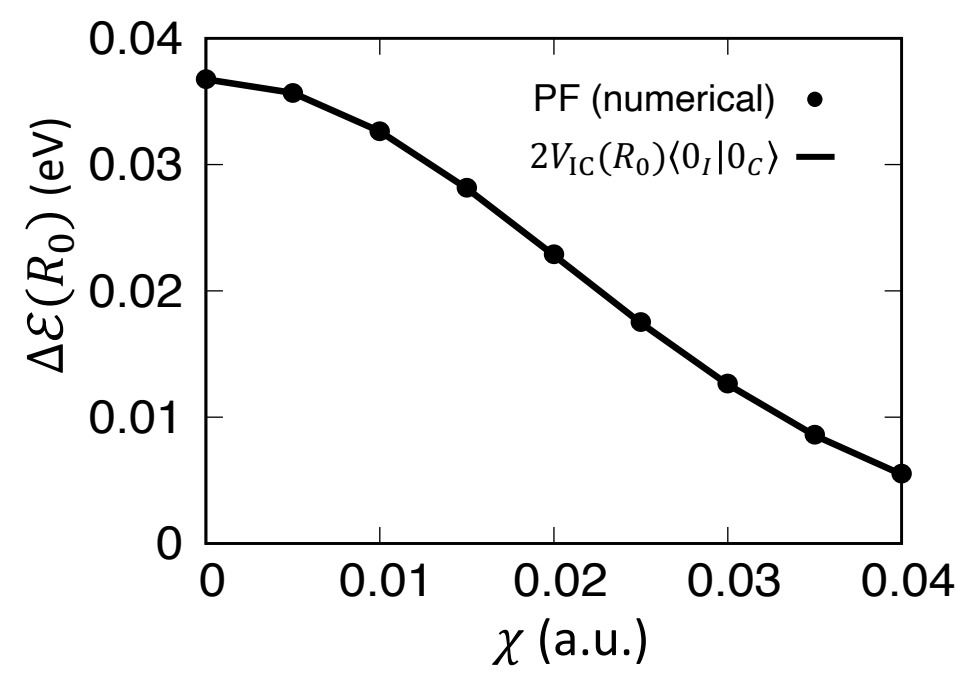

Figure S3: The energy splitting between the $\left|\Phi_{0}\right\rangle$ state and $\left|\Phi_{1}\right\rangle$ state at $R=R_{0} \approx 13.5$ a.u. (the molecular avoided crossing) as a function of $\chi$ with $\hbar \omega_{\mathrm{c}}=7.5 \mathrm{eV}$. The results are generated from the numerically exact solution of the PF Hamiltonian (dots), compared with the analytical expression (solid curve) of $2 V_{\mathrm{IC}}\left(R_{0}\right)\left\langle 0_{\mathrm{I}} \mid 0_{\mathrm{C}}\right\rangle$, where $\left\langle 0_{\mathrm{I}} \mid 0_{\mathrm{C}}\right\rangle=$ $\exp \left[-\frac{1}{2} \chi^{2}\left(\mu_{\mathrm{I}}-\mu_{\mathrm{C}}\right)^{2} /\left(\hbar \omega_{\mathrm{c}}\right)^{2}\right]$.

(state-dependent polarization). Note that we have explicitly ignored the contribution from $V_{\mathrm{IC}}(R)\left\langle 0_{\mathrm{I}} \mid n_{\mathrm{C}}\right\rangle$ for $n \geq 1$. The overlap $\left\langle 0_{\mathrm{I}} \mid 0_{\mathrm{C}}\right\rangle$ can be expressed analytically as follows

$$
\left\langle 0_{\mathrm{I}} \mid 0_{\mathrm{C}}\right\rangle=\exp \left[-\frac{1}{2} \frac{(\boldsymbol{\chi} \cdot \Delta \boldsymbol{\mu})^{2}}{\left(\hbar \omega_{\mathrm{c}}\right)^{2}}\right]
$$

It can be seen that $\Delta \mathcal{E}\left(R_{0}\right)$ and $2 V_{\mathrm{IC}}\left(R_{0}\right)\left\langle 0_{\mathrm{I}} \mid 0_{\mathrm{C}}\right\rangle$ are visually identical. This confirms our discussions in the main text and also indicates that the off-resonant contributions, i.e., $V_{\mathrm{IC}}(R)\left\langle 0_{\mathrm{I}} \mid n_{\mathrm{C}}\right\rangle$ for $n \geq 1$, plays a less important role in this energy splitting.

\section{Energy splittings at cavity induced avoided crossings}

Fig. S4 presents the energy splitting $\Delta \mathcal{E}\left(R_{i}\right)$ associated with three cavity-induced avoided crossings at $R_{1}, R_{2}$ and $R_{3}$ as a function of the light-matter coupling strength $\chi$, as we discussed in Fig. 2b from the main text. Here, we compare these energy-splittings that are computed numerically from the Pauli-Fierz Hamiltonian (filled circles) with the sim- 
ple analytical expression $2 V_{\mathrm{IC}}\left(R_{i}\right)\left\langle 0_{\mathrm{C}} \mid n_{\mathrm{I}}\right\rangle$ (solid lines), by ignoring all of the non-resonance contributions.

The overlaps $\left\langle 0_{\mathrm{C}} \mid n_{\mathrm{I}}\right\rangle$ are given analytically as

$$
\left\langle 0_{\mathrm{C}} \mid n_{\mathrm{I}}\right\rangle=\frac{1}{\sqrt{n !}}\left(\frac{\boldsymbol{\chi} \cdot \Delta \boldsymbol{\mu}}{\hbar \omega_{\mathrm{c}}}\right)^{n} e^{-\frac{1}{2}\left(\frac{\chi \cdot \Delta \mu}{\hbar \omega_{\mathrm{c}}}\right)^{2}}
$$

where $\Delta \boldsymbol{\mu}=\boldsymbol{\mu}_{\mathrm{I}}-\boldsymbol{\mu}_{\mathrm{C}}$, with the detailed expressions in Eqn. S39.

The avoided crossing at $R_{1}$ is mainly induced by the coupling between $|g, 1\rangle$ and $|e, 0\rangle$ states, through the molecule-cavity interactions from the transition dipole $\boldsymbol{\mu}_{e g}$. The energysplitting at this avoid crossing $\Delta \mathcal{E}\left(R_{1}\right)$ computed from the PF Hamiltonian or our analytical expression, for $\chi<0.004$ a.u. grows linearly with increasing $\chi$ which qualitatively as well as quantitatively matches with the prediction of the Rabi or JC model that ignores the permanent dipoles. Beyond this value, both Rabi (or the JC models) deviate from the prediction of the PF Hamiltonian as shown in Fig. 2b of the main text. This deviation of the energy gap computed from the Rabi model (or JC) and PF Hamiltonian is not due to the dipole self-energy of $\boldsymbol{\mu}_{e g}$, which added the same value for the polariton states $\left|\Phi_{1}\right\rangle$ and $\left|\Phi_{2}\right\rangle$. Rather, it is caused by the presence of permanent dipole moments $\boldsymbol{\mu}_{g g}$ and $\boldsymbol{\mu}_{e e}$ (and their associated DSE). These permanent dipoles cause couplings between $|g, n\rangle$ and $|g, n \pm 1\rangle$ states through $\left\langle g, n\left|\hat{\boldsymbol{\mu}}\left(\hat{a}^{\dagger}+\hat{a}\right)\right| g, n \pm 1\right\rangle$ (and similar couplings can also be found among $|e, n\rangle$ states). Hence, $|g, 1\rangle$ will couple to the $|g, 2\rangle$ states (and the $|g, 0\rangle$ state), the $|g, 2\rangle$ state couples to the $|g, 3\rangle$ state (and $|g, 1\rangle$ state), and so on and so forth.

For avoid crossing at $R_{1}$ under a smaller coupling strength, the effects from these permanent dipole moments are not as important as the effects from the transition dipole coupling $\left\langle g, 1\left|\hat{\boldsymbol{\mu}}\left(\hat{a}^{\dagger}+\hat{a}\right)\right| e, 0\right\rangle$, especially when the $|g, 1\rangle$ and $|e, 0\rangle$ states are energetically degenerate at the avoid crossing point. At a large coupling, all of these permanent dipole induced offresonance couplings are important, and hence the Rabi model is no longer accurate. Under the usual Fock state basis (vacuum's Fock states), it is not conceptually intuitive to discuss 


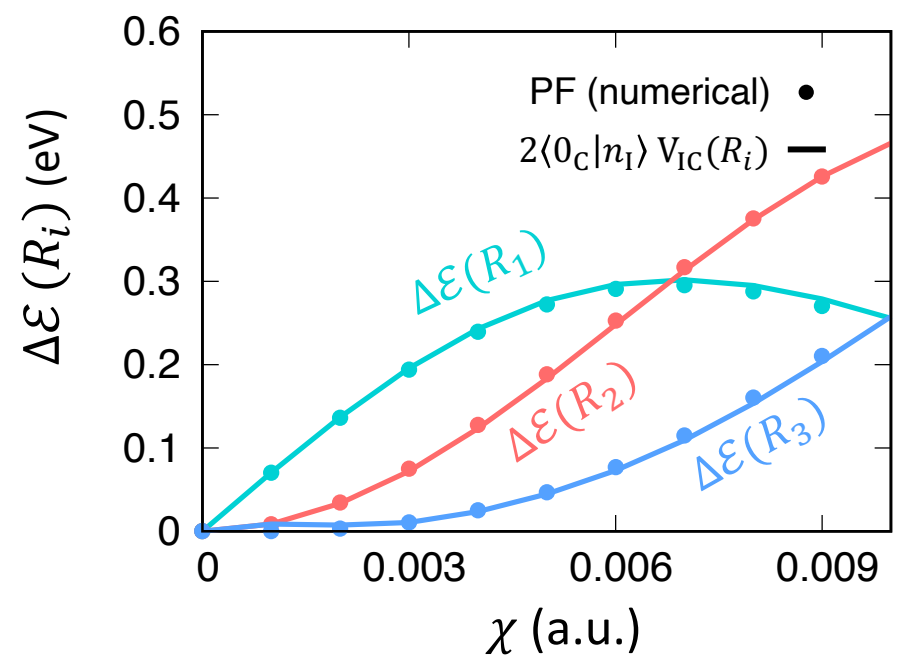

Figure S4: Energy splittings at cavity induced avoided crossings $\Delta \mathcal{E}\left(R_{i}\right)$ for $i \in 1,2,3$ at $R_{1}$ (green), $R_{2}$ (red) and $R_{3}$ (blue). These splittings are computed from the PF Hamiltonian (dots) and compared with the analytical expression $2 V_{\mathrm{IC}}\left(R_{i}\right)\left\langle n_{\mathrm{C}} \mid 0_{\mathrm{I}}\right\rangle$ (solid lines).

the role of $\boldsymbol{\mu}_{g g}$ and $\boldsymbol{\mu}_{e e}$. Under the polarized Fock state basis, on the other hand, it is more intuitive to understand these changes. This is because the splitting $\Delta \mathcal{E}\left(R_{1}\right)$ is mainly caused by the coupling $V_{\mathrm{IC}}\left\langle 0_{\mathrm{C}} \mid 1_{\mathrm{I}}\right\rangle$. By increasing $\boldsymbol{\chi} \cdot \hat{\boldsymbol{\mu}}$, the cavity induces a larger polarization difference between $\left|n_{\mathrm{C}}\right\rangle$ and $\left|m_{\mathrm{I}}\right\rangle$ (i.e., the displacement of two minima of the polariton surfaces along the photon coordinate $q$, see Fig. 1 of the main text), and the overlap between $\left|0_{\mathrm{C}}\right\rangle$ and $\left|1_{\mathrm{I}}\right\rangle$ first increase and then decrease. In fact, the numerical results of $\Delta \mathcal{E}\left(R_{1}\right)$ by numerically solving the PF Hamiltonian agree visually exactly with the analytical results from, $2 V_{\mathrm{IC}}\left(R_{1}\right)\left(\frac{\chi \cdot \Delta \mu}{\hbar \omega_{\mathrm{c}}}\right) \exp \left[-\frac{1}{2}\left(\frac{\chi \cdot \Delta \mu}{\hbar \omega_{\mathrm{c}}}\right)^{2}\right]$, as shown in Fig. S4.

A more drastic failure of the Rabi model comes from the crossing between state $|g, 2\rangle$ and $|e, 0\rangle$ at $R_{2}$, and between $|g, 3\rangle$ and $|e, 0\rangle$ at $R_{3}$. While the Rabi model does not predict any splitting, the exact solution for the PF Hamiltonian gives finite splitting. This is because that $\Delta \mathcal{E}\left(R_{2}\right)$ and $\Delta \mathcal{E}\left(R_{3}\right)$ are not caused by transition dipole $\boldsymbol{\mu}_{g e}$, due to the fact that $\left\langle g, n\left|\hat{\boldsymbol{\mu}}\left(\hat{a}^{\dagger}+\hat{a}\right)\right| e, m\right\rangle=\boldsymbol{\mu}_{g e}\left\langle n\left|\left(\hat{a}^{\dagger}+\hat{a}\right)\right| m\right\rangle=0$ for $m \neq n \pm 1$. Rather, these two splittings are caused by the coupling through both transition and permanent dipoles. For example, $|g, 2\rangle$ couples with $|g, 1\rangle$ through $\boldsymbol{\mu}_{g g}\left\langle 2\left|\left(\hat{a}^{\dagger}+\hat{a}\right)\right| 1\right\rangle$, and $|g, 1\rangle$ couples to the $|e, 0\rangle$ through $\boldsymbol{\mu}_{g e}\left\langle 1\left|\left(\hat{a}^{\dagger}+\hat{a}\right)\right| 0\right\rangle$, all together with other non-zero couplings. This, of course, should only be 
viewed as a qualitative picture to understand the splitting. To quantitatively calculate the splitting, one needs to include many Fock states to converge the results. In the polarized Fock state representation, on the other hand, these coupling can be simply and intuitively understood as $V_{\mathrm{IC}}\left\langle n_{\mathrm{C}} \mid m_{\mathrm{I}}\right\rangle$, that means $\Delta \mathcal{E}\left(R_{2}\right)=2 V_{\mathrm{IC}}\left\langle 0_{\mathrm{C}} \mid 2_{\mathrm{I}}\right\rangle$ and $\Delta \mathcal{E}\left(R_{3}\right)=2 V_{\mathrm{IC}}\left\langle 0_{\mathrm{C}} \mid 3_{\mathrm{I}}\right\rangle$ (and similar couplings can also be found among $|e, n\rangle$ states) when ignoring all of the other non-resonance couplings in the $\left|\alpha, n_{\alpha}\right\rangle$ basis, just significantly reduce the number of Fock states needed to obtain quantitative results, and at the same time, provide a conceptually intuitive picture to understand these splittings. In Fig. S4, we demonstrate that these simple analytic expressions provide visually indistinguishable results for $\Delta \mathcal{E}\left(R_{i}\right)$ compared to the numerically exact answer.

\section{Polaritonic Population Dynamics}

Fig. S5 presents the time-dependent polaritonic populations at various light-matter coupling strengths $\chi$ with the photon frequency $\hbar \omega_{\mathrm{c}}=1.5 \mathrm{eV}$. Fig. S6 presents the corresponding time-dependent photon populations. The initial state of the light-matter hybrid system is $|\Psi(t=0)\rangle \sim \exp \left(-\alpha\left(R-R_{g}\right)^{2}\right) \otimes\left|\Phi_{5}(R)\right\rangle$. Thus, at the initial time only polaritonic state $\left|\Phi_{5}(R)\right\rangle$ is populated. Near the Franck-Condon region, $\left|\Phi_{5}(R)\right\rangle \approx|\mathrm{C}, 0\rangle$ which corresponds to 0 photons in the cavity. As the polaritonic wavepacket travels towards the molecular avoided crossing at $R_{0}$, it encounters the light-induced avoided crossings at $R_{4}, R_{3}, R_{2}$ at $R_{1}$, see Fig. 2a of the main text.

At $\chi=0.001$ a.u., $\Delta \mathcal{E}\left(R_{4}\right), \Delta \mathcal{E}\left(R_{3}\right)$ and $\Delta \mathcal{E}\left(R_{2}\right)$ are negligible while $\Delta \mathcal{E}\left(R_{1}\right)$ is considerably large (see Fig.S4). Because of this, in Fig. S5a, a nearly complete polaritonic population transfer occurs from the $\left|\Phi_{5}(R)\right\rangle$ state to the $\left|\Phi_{4}(R)\right\rangle$ state, then from $\left|\Phi_{4}(R)\right\rangle$ to $\left|\Phi_{3}(R)\right\rangle$ and finally, from $\left|\Phi_{3}(R)\right\rangle$ to $\left|\Phi_{2}(R)\right\rangle$. Due to a larger splitting at $R_{1}$ (hence reducing the non-adiabaticity), the polaritonic transition from $\left|\Phi_{2}(R)\right\rangle$ to $\left|\Phi_{1}(R)\right\rangle$ is suppressed as $20 \%$ population remains in $\left|\Phi_{2}(R)\right\rangle$. Since the character of the $\left|\Phi_{2}(R)\right\rangle$ state beyond $R_{2}$ is 1photon character, this remaining population on $\left|\Phi_{2}(R)\right\rangle$ leads to the 1 photon probability 

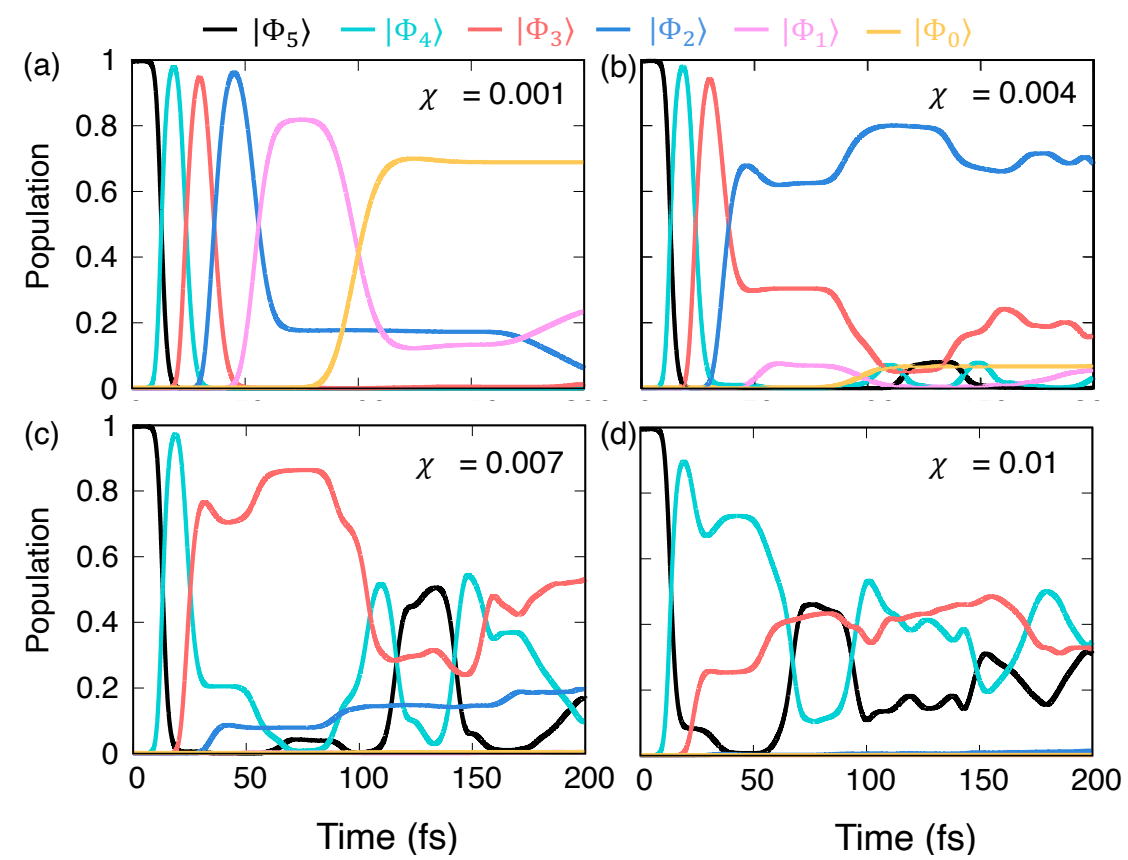

Figure S5: Polariton population dynamics at various light-matter couplings $\chi=0.001,0.004$, 0.007 and 0.01 a.u. and at $\hbar \omega_{\mathrm{c}}=1.5 \mathrm{eV}$.

as shown in Fig. S6a. The rest of the polaritonic population at $\left|\Phi_{1}(R)\right\rangle$ transfers to $\left|\Phi_{0}(R)\right\rangle$ through the molecular avoided crossing at $R_{0}$ and leads to dissociation.

At $\chi=0.004$ a.u., $\Delta \mathcal{E}\left(R_{4}\right)$ and $\Delta \mathcal{E}\left(R_{3}\right)$ remains negligible while $\Delta \mathcal{E}\left(R_{2}\right)$ and $\Delta \mathcal{E}\left(R_{1}\right)$ become considerably large. The polaritonic transition from $\left|\Phi_{3}(R)\right\rangle$ to $\left|\Phi_{2}(R)\right\rangle$ is suppressed, resulting in $25 \%$ population remains at $\left|\Phi_{3}(R)\right\rangle$ as shown in Fig. S5b, leading to the creation of 2-photon probability in Fig.S6b. The rest of polaritonic population $(\sim 75 \%)$ remains in the $\left|\Phi_{2}(R)\right\rangle$ state, as the non-adiabatic transition from $\left|\Phi_{2}(R)\right\rangle$ to $\left|\Phi_{1}(R)\right\rangle$ is strongly suppressed. Similar to the previous scenario, the $\left|\Phi_{2}(R)\right\rangle$ population leads to the creation of 1-photon probability. During the range of time presented here, only a small amount of population $(\sim 0.05)$ reaches to the ground polaritonic state $\left|\Phi_{0}(R)\right\rangle$.

At $\chi=0.007$, only $\Delta \mathcal{E}\left(R_{4}\right)$ is negligible. Due to the avoid crossing at $R_{3}$, part of polaritonic wavepacket travels to $\left|\Phi_{4}(R)\right\rangle$, creating 3-photon probability shown in Fig. S6c. The rest of the wavepacket transitions to $\left|\Phi_{3}(R)\right\rangle$ and remains there till $\sim 100 \mathrm{fs}$, as can be seen in Fig. S5c. This is because that the polaritonic transition from $\left|\Phi_{3}(R)\right\rangle$ to $\left|\Phi_{2}(R)\right\rangle$ 

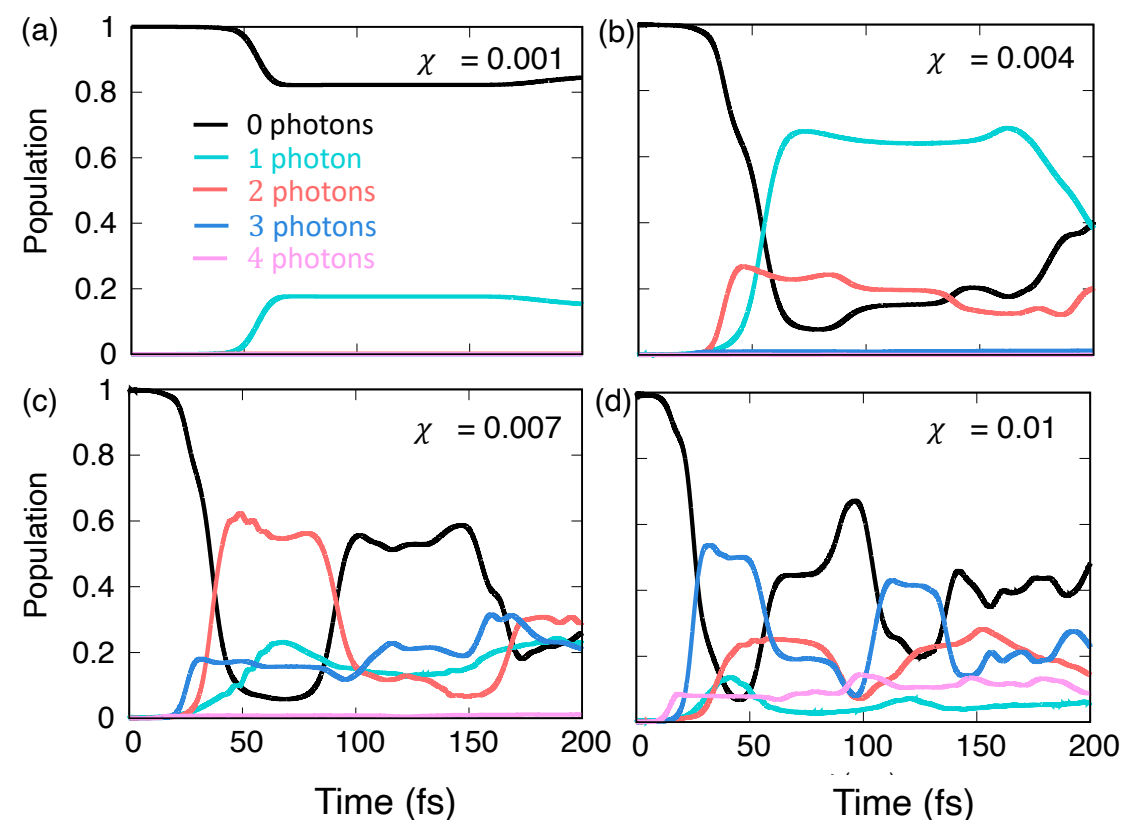

Figure S6: Photon population dynamics at various light-matter couplings $\chi=0.001,0.004$, 0.007 and 0.01 a.u. and at $\hbar \omega_{\mathrm{c}}=1.5 \mathrm{eV}$.

is significantly suppressed. The wavepacket then travels along the $\left|\Phi_{3}(R)\right\rangle$ surface, creating a substantial amount of 2-photon probability shown in Fig. S6c. At longer times as the wavepacket at $\left|\Phi_{3}(R)\right\rangle$ oscillates back and populates $\left|\Phi_{4}(R)\right\rangle$ and $\left|\Phi_{5}(R)\right\rangle$. Consequently, this leads to the re-absorption of the 2-photons to generate molecular excitation. This can be seen in Fig. S6c as the 2-photon probability decreases and 0-photon probability (here this corresponds to molecular excitation) increases around $100 \mathrm{fs}$.

Finally, at $\chi=0.01$, while all of the splittings reduces non-adiabatic transitions, $\Delta \mathcal{E}\left(R_{4}\right)$ is not large enough to significantly suppress the $\left|\Phi_{5}(R)\right\rangle$ to $\left|\Phi_{4}(R)\right\rangle$ transition. As a result, only a small portion ( $\sim 10 \%$ at $30-40$ fs, see Fig. S6d) of the wavepacket travels on the $\left|\Phi_{5}(R)\right\rangle$ surface leading to the creation of 4 photon probability in Fig.S6d. Meanwhile, a large $\Delta \mathcal{E}\left(R_{3}\right)$ significantly suppress $\left|\Phi_{4}(R)\right\rangle$ to $\left|\Phi_{3}(R)\right\rangle$ transition, and as a result for $t<100$ fs mainly $\left|\Phi_{4}(R)\right\rangle$ remains populated while some population is leaked into $\left|\Phi_{3}(R)\right\rangle$ as can be seen in Fig.S5d. Similar to the previous cases, the nuclear wavepacket on $\left|\Phi_{4}(R)\right\rangle$ leads to a signification amount of creation of 3-photon probability in Fig. S6d. At longer times, 
the wavepacket oscillates back and forth, making the transition from $\left|\Phi_{4}(R)\right\rangle$ to $\left|\Phi_{5}(R)\right\rangle$ and vice-versa, leading to the oscillatory populations of $\left|\Phi_{4}(R)\right\rangle$ and $\left|\Phi_{5}(R)\right\rangle$. Meanwhile, this oscillation of the polaritonic wavepacket results in absorption and emission of 3-photon probability as shown in Fig. S6d.

\section{Generalized theory of the polarized Fock States}

In the main text, we have presented the polarized Fock States representation for a two-state molecular system and explicitly assumed that the eigenstates of the dipole operator $\hat{\boldsymbol{\mu}}$ are diabatic, such that their character does not depend on nuclear positions. Here, we present the most general version of the PFS without assuming that they are diabatic states.

For a molecular system, it is convenient to obtain its adiabatic states, $\{|i(\mathbf{R})\rangle\}$, which is the eigenstates of the electronic Hamiltonian $\hat{H}_{\mathrm{M}}-\hat{T}_{\mathbf{R}}$, where $\hat{H}_{\mathrm{M}}$ is the matter Hamiltonian and $\hat{T}_{\mathbf{R}}$ is the nuclear kinetic energy operator. Note that $|i(\mathbf{R})\rangle$ parametrically dependents on $\mathbf{R}$. The molecular Hamiltonian in the adiabatic representation is given as follows

$$
\hat{H}_{\mathrm{M}}=\frac{1}{2 M}\left(\hat{\mathbf{P}}-i \hbar \sum_{i, j}\left\langle i\left|\nabla_{\mathbf{R}}\right| j\right\rangle|i\rangle\langle j|\right)^{2}+\sum_{i} E_{i}(\mathbf{R})|i\rangle\langle i|
$$

The dipole moment operator $\hat{\mu}$ in the adiabatic basis is

$$
\hat{\mu}=\sum_{i, j} \mu_{i, j}(\mathbf{R})|i(\mathbf{R})\rangle\langle j(\mathbf{R})|
$$

These information in Eqn. S46-S47 can be conveniently obtained from modern electronic structure calculations. We obtain the eigenstates of the dipole moment operator $\hat{\mu}$ (in this adiabatic subspace) using the unitary operator $\hat{U}_{\mu}$ through the following relations

$$
\hat{U}_{\mu}^{\dagger} \hat{\mu} \hat{U}_{\mu}=\sum_{\alpha} \mu_{\alpha}(\mathbf{R})|\alpha(R)\rangle\langle\alpha(\mathbf{R})|
$$

where $\hat{U}_{\mu}$ can be obtained from the standard diagonalization procedure. Note that $\{|\alpha(\mathbf{R})\rangle\}$ 
is a good approximation of diabatic states, and this procedure is referred to as the MullikenHush diabatization. ${ }^{9-13}$ Here, we do not explicitly assume that they are $R$-independent (i.e., treating them as strict diabatic states). Under the $\{|\alpha\rangle\}$ representation, $\hat{H}_{\mathrm{M}}$ is given as

$$
\hat{H}_{\mathrm{M}}=\frac{1}{2 M}\left(\hat{\mathbf{P}}-i \hbar \sum_{\alpha, \beta}\left\langle\alpha\left|\nabla_{\mathbf{R}}\right| \beta\right\rangle|\alpha\rangle\langle\beta|\right)^{2}+\sum_{\alpha} V_{\alpha \alpha}(\mathbf{R})|\alpha\rangle\left\langle\alpha\left|+\sum_{\alpha \neq \beta} V_{\alpha \beta}(\mathbf{R})\right| \alpha\right\rangle\langle\beta|,
$$

where $V_{\alpha \beta}(\mathbf{R})=\left\langle\alpha\left|\hat{H}_{\mathrm{M}}-\hat{T}_{\mathbf{R}}\right| \beta\right\rangle$ is the electronic Hamiltonian's matrix elements.

The Pauli-Fierz (PF) non-relativistic QED Hamiltonian (Eqn. 1 in the main text) in the polarized-Fock state $\left|\alpha(\mathbf{R}), n_{\alpha}(\mathbf{R})\right\rangle \equiv|\alpha(\mathbf{R})\rangle \otimes\left|n_{\alpha}(\mathbf{R})\right\rangle$ (see its definition in the main text) is expressed as

$$
\begin{aligned}
\hat{H} & =\frac{1}{2 M}\left(\hat{\mathbf{P}}-i \hbar \sum_{\alpha, \beta} \sum_{n_{\alpha}, m_{\beta}} d_{n_{\alpha}, m_{\beta}}^{\alpha, \beta}\left|\alpha, n_{\alpha}\right\rangle\left\langle\beta, m_{\beta}\right|\right)^{2} \\
& +\sum_{\alpha} \sum_{n_{\alpha}}\left(V_{\alpha \alpha}(R)+\left(n_{\alpha}+\frac{1}{2}\right) \hbar \omega_{c}\right)\left|\alpha, n_{\alpha}\right\rangle\left\langle\alpha, n_{\alpha}\right| \\
& +\sum_{\alpha \neq \beta} \sum_{n_{\alpha}, m_{\beta}} V_{\alpha \beta}(\mathbf{R})\left\langle n_{\alpha} \mid m_{\beta}\right\rangle\left|\alpha, n_{\alpha}\right\rangle\left\langle\beta, m_{\beta}\right|,
\end{aligned}
$$

where the non-adiabatic coupling is

$$
d_{n_{\alpha}, m_{\beta}}^{\alpha, \beta} \equiv\left\langle\alpha, n_{\alpha}\left|\nabla_{\mathbf{R}}\right| \beta, m_{\beta}\right\rangle=\left\langle\alpha\left|\nabla_{\mathbf{R}}\right| \beta\right\rangle\left\langle n_{\alpha} \mid m_{\beta}\right\rangle+\left\langle n_{\alpha}\left|\nabla_{\mathbf{R}}\right| m_{\beta}\right\rangle \delta_{\alpha, \beta} .
$$

Under the assumption that $\left\langle\alpha\left|\nabla_{\mathbf{R}}\right| \beta\right\rangle=0$, that is, $\{|\alpha\rangle,|\beta\rangle\}$ is the strict diabatic basis, the Hamiltonian in Eqn. S50 reduces to the Hamiltonian under the PFS representation in the main text.

Further, we note that in this generalized scenario, the $|\alpha\rangle$ state with $n_{\alpha}$ photons and the $|\beta\rangle$ state with $m_{\beta}$ photons are coupled to each other through the scaled couplings $V_{\alpha, \beta}(\mathbf{R})\left\langle n_{\alpha} \mid m_{\beta}\right\rangle$ as well as the non-adiabatic couplings $\left\langle\alpha\left|\nabla_{\mathbf{R}}\right| \beta\right\rangle\left\langle n_{\alpha} \mid m_{\beta}\right\rangle$. These terms will cause quantum transitions that can be exploited to perform downconversion as shown in the main-text. We also note that with an increasing light-matter coupling the overlap $\left\langle 0_{\alpha} \mid 0_{\beta}\right\rangle$ 
falls off as a Gaussian function (when $\mu_{\alpha}(\mathbf{R}) \neq \mu_{\beta}(\mathbf{R})$ ). This will result in a suppression of quantum transitions between $\left|\alpha, 0_{\alpha}\right\rangle$ and $\left|\beta, 0_{\beta}\right\rangle$, as both $V_{\alpha, \beta}(\mathbf{R})\left\langle 0_{\alpha} \mid 0_{\beta}\right\rangle$ and $\left\langle\alpha\left|\nabla_{\mathbf{R}}\right| \beta\right\rangle\left\langle 0_{\alpha} \mid 0_{\beta}\right\rangle$ are scaled down. Hence, increasing light-matter coupling strength will enhance the nonadiabatic transition between polaritonic states that are formed by hybridizing $\left|\alpha, 0_{\alpha}\right\rangle$ and $\left|\beta, 0_{\beta}\right\rangle$. This is essentially what we demonstrated by enhancing photodissociation in the main text (where we have explicitly assumed that $\left\langle\alpha\left|\nabla_{\mathbf{R}}\right| \beta\right\rangle=0$ ). Therefore, even at the presence of finite $\left\langle\alpha\left|\nabla_{\mathbf{R}}\right| \beta\right\rangle$, i.e., when Mulliken-Hush diabatic states are no longer strict diabatic states, all effect shown in the main text are expected to occur.

\section{Polarized Fock States in the infinite electronic basis limit}

While it may not be practically useful to consider infinite electronic states, it is an interesting theoretical limit. The dipole operator for the molecule is defined as $\hat{\boldsymbol{\mu}}=\sum_{j} z_{j} \hat{r}_{j}+\sum_{l} z_{l} \hat{R}_{l}$, where the sum over $j$ includes all electrons and the sum over $l$ includes all nuclei. When considering the complete electronic state limit (adiabatic electronic states, hence also the MH diabatic states) $\{|\alpha(\mathbf{R})\rangle\}$, the dipole operator at a particular nuclear position $\mathbf{R} \equiv\left\{R_{l}\right\}$ is expressed as follows

$$
\hat{\boldsymbol{\mu}}(\mathbf{R})=\sum_{\alpha}\langle\alpha(\mathbf{R})|\hat{\boldsymbol{\mu}}(\mathbf{R})| \alpha(\mathbf{R})\rangle|\alpha(\mathbf{R})\rangle\langle\alpha(\mathbf{R})|=\sum_{k} z_{k} \hat{r}_{k}+\sum_{l} z_{l} R_{l}
$$

Under the infinite basis limit, the eigenstates of $\hat{\boldsymbol{\mu}}(\mathbf{R})$ becomes the eigenstates of the eletronic position operator $\hat{\mathbf{r}}$, such that $\hat{\boldsymbol{\mu}}(\mathbf{R})|\mathbf{r}\rangle=\boldsymbol{\mu}(\mathbf{R}, \mathbf{r})|\mathbf{r}\rangle$, where $\boldsymbol{\mu}(\mathbf{R}, \mathbf{r})=\sum_{k} z_{k} r_{k}+\sum_{l} z_{l} R_{l}$. Hence, these MH electronic diabatic states becomes positon operator's eigenstates. It is often convenient to use grid basis to represent electronic position operators' eigenstate, and we denote these grid basis as $\left\{\left|\mathbf{r}^{i}\right\rangle\right\} \equiv\left\{\left|r_{1}^{i}\right\rangle \ldots \otimes\left|r_{k}^{i}\right\rangle \otimes \ldots\right\}$, where $i$ is label of the grid basis for the electronic coordinates, and the eigenequation of $\hat{\boldsymbol{\mu}}(\mathbf{R})$ becomes $\hat{\boldsymbol{\mu}}(\mathbf{R})\left|\mathbf{r}^{i}\right\rangle=\boldsymbol{\mu}\left(\mathbf{R}, \mathbf{r}^{i}\right)\left|\mathbf{r}^{i}\right\rangle$.

As a result, the MH electronic-PFS basis $\left\{\left|\alpha, n_{\alpha}\right\rangle\right\}$ becomes $\left\{\left|\mathbf{r}^{i}, n_{i}(\mathbf{R})\right\rangle\right\} \equiv\left\{\left|\mathbf{r}^{i}\right\rangle \otimes\right.$ $\left.\left|n\left(\mathbf{r}^{i}, \mathbf{R}\right)\right\rangle\right\}$, where $\left|n\left(\mathbf{r}^{i}, \mathbf{R}\right)\right\rangle$ is the polarized Fock state associated with a reference elec- 
tronic coordinate $\mathbf{r}^{i}$. Hence, PFS does not parametrically depend on electronic coordinates (because it only depends on a particular electronic configuration $\mathbf{r}^{i}$, but it still parametrically depends on the nuclear configuration $\mathbf{R}$. The polaritonic Hamiltonian $\hat{H}_{\mathrm{pl}} \equiv \hat{H}-\hat{T}_{R}$ in the $\left\{\left|\mathbf{r}_{i}, n_{i}(\mathbf{R})\right\rangle\right\}$ basis is expressed as follows

$\hat{H}_{\mathrm{pl}}=\sum_{i, j, n_{i}, m_{j}}\left\langle n_{i}(\mathbf{R}) \mid m_{j}(\mathbf{R})\right\rangle\left\langle\mathbf{r}^{i}\left|\hat{H}_{\mathrm{el}}\right| \mathbf{r}^{j}\right\rangle\left|\mathbf{r}^{i}, n_{i}(\mathbf{R})\right\rangle\left\langle\mathbf{r}^{j}, m_{j}(\mathbf{R})\left|+\sum_{n_{i}}\left(n_{i}+\frac{1}{2}\right) \hbar \omega_{c}\right| n_{i}(\mathbf{R})\right\rangle\left\langle n_{i}(\mathbf{R})\right|$,

where $\hat{H}_{\mathrm{el}}=\hat{H}_{\mathrm{M}}-\hat{T}_{R}$.

Even under this limit, $\hat{H}_{\mathrm{pl}}$ in Eq. S53 is still fundamentally different than the one introduced in Ref 14 which uses polarized vacuum states $\{|n(\mathbf{r}, \mathbf{R})\rangle\}$. These polarized vacuum states are defined ${ }^{14}$ as follows

$$
\frac{1}{2} \hat{p}^{2}+\frac{1}{2} \omega_{\mathrm{c}}^{2}\left(\hat{q}+\sqrt{\frac{2}{\hbar \omega_{\mathrm{c}}^{3}}} \chi \cdot \mu(\mathbf{r}, \mathbf{R})\right)^{2}|n(\mathbf{r}, \mathbf{R})\rangle=\left(n+\frac{1}{2}\right) \hbar \omega_{\mathrm{c}}|n(\mathbf{r}, \mathbf{R})\rangle
$$

where $\mu(\mathbf{r}, \mathbf{R})=\sum_{k} z_{k} r_{k}+\sum_{l} z_{l} R_{l}$ is the dipole operator at a particular nuclear configuration $\mathbf{R}$ and at a particular electronic configuration $\mathbf{r}$. Unlike the PFS introduced in this work, these polarized vacuum states ${ }^{14}$ parametrically depend on both the electronic coordinates $\mathbf{r}$ and the nuclear coordinates $\mathbf{R}$.

If one uses the grid basis $\left|\mathbf{r}^{i}\right\rangle$ to represent electronic coordinates $\hat{\mathbf{r}}$ and use the polarized vacuum states $\{|n(\mathbf{r}, \mathbf{R})\rangle\}$ defined in the above equation, the polaritonic Hamiltonian in the $\left\{\left|\mathbf{r}^{i}, n(\mathbf{r}, \mathbf{R})\right\rangle\right\}$ basis can be expressed as

$$
\begin{aligned}
\hat{H}_{\mathrm{pl}} & =\sum_{i, j, n, m}\left\langle\mathbf{r}^{i}, n(\mathbf{r}, \mathbf{R})\left|\hat{H}_{\mathrm{el}}\right| \mathbf{r}^{j}, m(\mathbf{r}, \mathbf{R})\right\rangle\left|\mathbf{r}^{i}, n(\mathbf{r}, \mathbf{R})\right\rangle\left\langle\mathbf{r}^{j}, m(\mathbf{r}, \mathbf{R})\left|+\sum_{n}\left(n+\frac{1}{2}\right) \hbar \omega_{\mathrm{c}}\right| n(\mathbf{r}, \mathbf{R})\right\rangle\langle n(\mathbf{r}, \mathbf{R})| \\
& =\sum_{i, j}\left\langle\mathbf{r}^{i}\left|\left(\frac{1}{2 m_{e}}\left(\hat{\mathbf{p}}_{\mathrm{r}}-i \hbar \mathbf{d}_{\mathbf{r}}\right)^{2}+\hat{V}_{\text {coul }}\right)\right| \mathbf{r}^{j}\right\rangle\left|\mathbf{r}^{i}\right\rangle\left\langle\mathbf{r}^{j}\left|+\sum_{n}\left(n+\frac{1}{2}\right) \hbar \omega_{c}\right| n(\mathbf{r}, \mathbf{R})\right\rangle\langle n(\mathbf{r}, \mathbf{R})|
\end{aligned}
$$


where $\hat{V}_{\text {coul }}=\hat{H}_{\mathrm{M}}-\hat{T}_{\mathbf{R}}-\hat{T}_{\mathbf{r}}$ is the coulomb potential operator. This is essentially the expression of $\hat{H}_{\mathrm{pl}}$ derived in Ref. ${ }^{14}$ (Eq. 18 and 24 in that work). Further, $\mathbf{d}_{\mathbf{r}}=\sum_{n m}\left\langle m(\mathbf{r}, \mathbf{R})\left|\nabla_{\mathbf{r}}\right| n(\mathbf{r}, \mathbf{R})\right\rangle$ $\times|m(\mathbf{r}, \mathbf{R})\rangle\langle n(\mathbf{r}, \mathbf{R})|$ is the electronic derivative coupling among the polarized vacuum states $\{|n(\mathbf{r}, \mathbf{R})\rangle\}$, originated from their parametric dependence of electronic position $\mathbf{r}$.

It can be clearly seen that the basis used in Eqn. S53 (with PFS introduced in this work) and in Eqn. S55 (and the polarized vacuum states introduced in Ref. 14) are fundamentally different as follows (i) the PFS used in this work does not carry any parametric dependence of $\mathbf{r}$ and therefore the electronic derivative couplings $\mathbf{d}_{\mathbf{r}}$ do not appear in Eqn. S53 (or Eqn. 12 in the main text) when using the truncated electronic subspace, whereas using polarized vacuum state do give $\mathbf{d}_{\mathbf{r}}$, (ii) Polarized Fock states are non-orthogonal to each other, and therefore it requires one to compute the photonic overlap integral $\left\langle m_{j}(\mathbf{R}) \mid n_{i}(\mathbf{R})\right\rangle$ to evaluate $\hat{H}_{\mathrm{pl}}$; in contrast, the polarized vacuum states in Ref 14 are orthogonal to each other, such that $\langle m(\mathbf{r}, \mathbf{R}) \mid n(\mathbf{r}, \mathbf{R})\rangle=\delta_{n m}$, and (iii) unlike the PFS representation which requires one to compute dipole moment matrix elements, the polarized vacuum states in Ref. 14 requires computing the velocity matrix elements of the electronic degrees freedom (same as in the "p.A" Hamiltonian), and therefore may subject to erroneous result when truncating the electronic subspace to few levels, which has been extensively discussed in the Coulomb gauge Hamiltonian. ${ }^{3,15,16}$

\section{Derivation of the Pauli-Fierz Hamiltonian}

We provide a brief derivation of the commonly used Pauli-Fierz non-relativistic Hamiltonian $^{14,17}$ for light-matter interactions. We begin by writing the electric field as $\mathbf{E}(\mathbf{r})=$ $\mathbf{E}_{\|}(\mathbf{r})+\mathbf{E}_{\perp}(\mathbf{r})$, with its longitudinal part $\mathbf{E}_{\|}(\mathbf{r})$ that is curl free (irrotational) $\nabla \times \mathbf{E}_{\|}(\mathbf{r})=0$, and the transverse part that is divergence free (solenoidal) $\nabla \cdot \mathbf{E}_{\perp}(\mathbf{r})=0$. The magnetic field is purely transverse $\mathbf{B}(\mathbf{r})=\mathbf{B}_{\perp}(\mathbf{r})$, because $\nabla \cdot \mathbf{B}(\mathbf{r})=0$.

By choosing the Coulomb Gauge $\nabla \cdot \mathbf{A}=0$ that makes the vector potential purely transverse, the Hamiltonian of $N$ point charge particles (including both electrons and nuclei) 
can be written as follows

$$
\hat{H}=\sum_{i}^{N} \frac{\left(\mathbf{p}_{i}-q_{i} \mathbf{A}_{\perp}\left(\mathbf{r}_{i}\right)\right)^{2}}{2 m_{i}}+\frac{\epsilon_{0}}{2} \int d r^{3} \mathbf{E}_{\|}^{2}(\mathbf{r})+\frac{\epsilon_{0}}{2} \int d r^{3}\left[\mathbf{E}_{\perp}^{2}(\mathbf{r})+c^{2} \mathbf{B}_{\perp}^{2}(\mathbf{r})\right]
$$

where the sum includes both the nuclear and electronic DOFs, $\mathbf{r}_{i}$ and $\mathbf{p}_{i}$ are the position and momentum of the charged particle $i$, with the charge $q_{i}$ and mass $m_{i}$. Further, $\mathbf{A}_{\perp}(\mathbf{r}), \mathbf{E}_{\perp}(\mathbf{r})$ and $\mathbf{B}_{\perp}(\mathbf{r})$ are the transverse vector potential, electric field, and magnetic field, respectively. The energy associated with $\mathbf{E}_{\|}(\mathbf{r})$ (the second term in Eqn. S56) is given by

$$
\begin{aligned}
& \frac{\epsilon_{0}}{2} \int d r^{3} \mathbf{E}_{\|}^{2}(\mathbf{r})=\sum_{i} \frac{q_{i}^{2}}{2 \epsilon_{0}(2 \pi)^{3}} \int \frac{d k^{3}}{k^{2}}+\frac{1}{8 \pi \epsilon_{0}} \sum_{i \neq j} \frac{q_{i} q_{j}}{\left|r_{i}-r_{j}\right|} \\
& =\sum_{i} \epsilon_{i}^{\infty}+V_{\text {coul }}(\mathbf{r}) \equiv V_{\text {coul }}(\mathbf{r}) .
\end{aligned}
$$

Here, the first term $\sum_{i} \epsilon_{i}^{\infty}$ in the second line of Eqn. S57 is a time-independent infinite quantity that is referred to as the self-energy (not to be confused with the dipole self-energy), which can be regarded as a shift of the zero-point energy ${ }^{18}$ and is dropped in the last line of the above equation. In short, the coulomb potential $V_{\text {coul }}(\mathbf{r})$ emerges from the longitudinal electric field. The last term in Eqn. S56 is the energy associated with the transverse fields $\mathbf{E}_{\perp}(\mathbf{r})$ and $\mathbf{B}_{\perp}(\mathbf{r}) . \mathbf{A}_{\perp}(\mathbf{r}), \mathbf{E}_{\perp}(\mathbf{r})$ and $\mathbf{B}_{\perp}(\mathbf{r})$ satisfy the boundary conditions of an arbitrary cubic box of length $L \rightarrow \infty$ for vacuum, ${ }^{18,19}$ and are quantized ${ }^{18,19}$ as follows

$$
\begin{aligned}
& \mathbf{A}_{\perp}(\mathbf{r})=\sum_{j} \frac{\boldsymbol{\chi}_{j}}{\omega_{j}}\left(\hat{a}_{j} e^{i \mathbf{k}_{j} \cdot \mathbf{r}}+\hat{a}_{j}^{\dagger} e^{-i \mathbf{k}_{j} \cdot \mathbf{r}}\right) \\
& \mathbf{E}_{\perp}(\mathbf{r})=i \sum_{j} \boldsymbol{\chi}_{j}\left(\hat{a}_{j} e^{i \mathbf{k}_{j} \cdot \mathbf{r}}-\hat{a}_{j}^{\dagger} e^{-i \mathbf{k}_{j} \cdot \mathbf{r}}\right) \\
& \mathbf{B}_{\perp}(\mathbf{r})=i \sum_{j} \mathbf{k}_{j} \times \boldsymbol{\chi}_{j}\left(\hat{a}_{j} e^{i \mathbf{k}_{j} \cdot \mathbf{r}}-\hat{a}_{j}^{\dagger} e^{-i \mathbf{k}_{j} \cdot \mathbf{r}}\right)
\end{aligned}
$$

where, $\hat{\mathbf{e}}_{j} \sqrt{\frac{\hbar \omega_{j}}{2 \varepsilon_{0} \mathcal{V}}}=\boldsymbol{\chi}_{j}$. Here, $j$ is the index of the quantized radiational mode, $\hat{a}_{j}^{\dagger}$ and $\hat{a}_{j}$ are the creation and annihilation operators of photon, respectively, the frequency for mode $j$ is 
$\omega_{j}=c\left|\mathbf{k}_{j}\right|=j \pi c / L$. Here $c$ is the speed of light, and $j \geq 1$ is an integer.

When charged particles are placed in a planar Fabry-Pérot (FB) microcavity, only those radiation modes with wave vector $\mathbf{k}_{j}$ that are perpendicular to the mirror surfaces and satisfy the boundary condition of the cavity (i.e. L is taken as the distance between the mirrors) are important. Therefore, we only consider $\mathbf{k}_{j}=\left|\mathbf{k}_{j}\right| \hat{\mathbf{k}}$ which align in the direction of the unit vector $\hat{\mathbf{k}}$ that is perpendicular to the mirror surfaces and $\hat{\boldsymbol{\epsilon}}_{j} \perp \hat{\mathbf{k}}$ is the polarization unit vector for $\mathbf{E}_{\perp}(\mathbf{r})$.

Using the above expressions, the energy of the transverse fields, i.e., the last term in Eqn. S56 is quantized as follows

$$
\frac{\varepsilon_{0}}{2} \int_{\mathcal{V}} d r^{3}\left[\mathbf{E}_{\perp}^{2}(\mathbf{r})+c^{2} \mathbf{B}_{\perp}^{2}(\mathbf{r})\right]=\sum_{j}\left(\hat{a}_{j}^{\dagger} \hat{a}_{j}+\frac{1}{2}\right) \hbar \omega_{j},
$$

where the spatial integral $d r^{3}$ is done within the effective quantized volume $\mathcal{V}$ of the FB cavity.

Assuming that the dimension of the molecular system is much smaller than the length of the cavity, which is commonly referred to as the long wave-length approximation, the transverse fields can be treated as it is spatially uniform, i.e., $e^{i \mathbf{k}_{j} \cdot \mathbf{r}} \approx 1$, such that

$$
\mathbf{A}_{\perp}(\mathbf{r}) \approx \mathbf{A}_{\perp}=\sum_{j} \frac{\chi_{j}}{\omega_{j}}\left(\hat{a}_{j}+\hat{a}_{j}^{\dagger}\right)
$$

With the above approximation, the first term in Eqn. S56 is expressed as

$$
\sum_{i}^{N} \frac{\left(\mathbf{p}_{i}-q_{i} \mathbf{A}_{\perp}\left(\mathbf{r}_{i}\right)\right)^{2}}{2 m_{i}} \approx \sum_{i}^{N} \frac{\left(\mathbf{p}_{i}-q_{i} \mathbf{A}_{\perp}\right)^{2}}{2 m_{i}}
$$

Further, we consider only a single mode of the radiation field along the $\hat{\boldsymbol{\epsilon}}$ direction (which is commonly referred to as the single mode approximation in cavity QED), with the frequency $\omega_{\mathrm{c}}=\pi c / L\left(c\right.$ is the speed of the light, and $\omega_{\mathrm{c}}$ represents the single mode frequency of the cavity), and the corresponding photonic creation and annihilation operators $\hat{a}^{\dagger}$ and $\hat{a}$. The 
single mode cavity-QED Hamiltonian under the long-wavelength limit in the Coulomb Gauge is then given as

$$
\hat{H}_{\mathrm{p} \cdot \mathrm{A}}=\sum_{i}^{N} \frac{1}{2 m_{i}}\left[\mathbf{p}_{i}-\frac{q_{i} \boldsymbol{\chi}}{\omega_{\mathrm{c}}}\left(\hat{a}+\hat{a}^{\dagger}\right)\right]^{2}+V_{\text {coul }}(\mathbf{r})+\left(\hat{a}^{\dagger} \hat{a}+\frac{1}{2}\right) \hbar \omega_{\mathrm{c}}
$$

The above Hamiltonian is referred to as the "p $\cdot A$ " Hamiltonian. The above Hamiltonian can be expressed in terms of photonic position $\hat{q}=\sqrt{\hbar / 2 \omega_{\mathrm{c}}}\left(\hat{a}^{\dagger}+\hat{a}\right)$ and photonic momentum operator $\hat{p}=i \sqrt{\hbar \omega_{\mathrm{c}} / 2}\left(\hat{a}^{\dagger}-\hat{a}\right)$ as

$$
\hat{H}_{\mathrm{p} \cdot \mathrm{A}}=\sum_{i}^{N} \frac{1}{2 m_{i}}\left[\mathbf{p}_{i}-\frac{q_{i} \hat{\boldsymbol{\epsilon}}}{\omega_{\mathrm{c}}} \sqrt{\frac{\hbar \omega_{\mathrm{c}}}{2 \varepsilon_{0} \mathcal{V}}}\left(\hat{a}+\hat{a}^{\dagger}\right)\right]^{2}+V_{\text {coul }}(\mathbf{r})+\left(\hat{a}^{\dagger} \hat{a}+\frac{1}{2}\right) \hbar \omega_{\mathrm{c}}
$$

To arrive at the convenient " $\mathrm{d} \cdot \mathrm{E}$ " form of the QED Hamiltonian, ${ }^{18,19}$ we use the following unitary transformation, which is commonly referred to as the Power-Zienau-Woolley (PZW) Gauge transformation ${ }^{17,19}$

$$
\hat{U}_{\mathrm{pzw}}=\exp \left[\frac{i}{\hbar} \mathbf{A}_{\perp} \cdot \hat{\boldsymbol{\mu}}(\mathbf{r})\right]
$$

Here, $\hat{\boldsymbol{\mu}}(\mathbf{r})=\sum_{i} q_{i} \mathbf{r}_{i}$ represents the total dipole operator of the molecular system (with contributions from both electrons and nuclei). Note, $\hat{U}_{\mathrm{pzw}}$ is essentially a boost operator for the particle momentum $\mathbf{p}_{i}$ such that:

$$
U_{\mathrm{pzw}}^{\dagger}\left(\mathbf{p}_{i}-q_{i} \mathbf{A}_{\perp}\right) U_{\mathrm{pzw}} \rightarrow \mathbf{p}_{i} .
$$

The above expression can be verified by using the Baker-Hausdorff identity $e^{\hat{A}} \hat{B} e^{-\hat{A}}=\hat{B}+$ $[\hat{A}, \hat{B}]+\frac{1}{2 !}[\hat{A},[\hat{A}, \hat{B}]]+\ldots$, with $\hat{A} \equiv \frac{i}{\hbar} \mathbf{A}_{\perp} \cdot \hat{\boldsymbol{\mu}}(\mathbf{r})$ and $\hat{B} \equiv \mathbf{p}_{i}-q_{i} \mathbf{A}_{\perp}$. Using Eqn. S65, we obtain

$$
U_{\mathrm{pzw}}^{\dagger}\left[\sum_{i}^{N} \frac{\left(\mathbf{p}_{i}-q_{i} \mathbf{A}_{\perp}\right)^{2}}{2 m_{i}}\right] U_{\mathrm{pzw}} \rightarrow \sum_{i}^{N} \frac{1}{2 m_{i}}\left[U_{\mathrm{pzw}}^{\dagger}\left(\mathbf{p}_{i}-q_{i} \mathbf{A}_{\perp}\right) U_{\mathrm{pzw}}\right]^{2}=\sum_{i}^{N} \frac{\mathbf{p}_{i}^{2}}{2 m_{i}}
$$

Meanwhile, the coulomb potential $V_{\text {coul }}(\mathbf{r})$ remains invariant upon the PZW transformation 
is

$$
U_{\mathrm{pzW}}^{\dagger}\left[V_{\mathrm{coul}}(\mathbf{r})\right] U_{\mathrm{pzw}} \rightarrow V_{\mathrm{coul}}(\mathbf{r})
$$

Using the Baker-Hausdorff identity, the last term in the Eqn. S62, i.e., the energy of the transverse fields, is transformed as

$U_{\mathrm{pzw}}^{\dagger}\left[\left(\hat{a}^{\dagger} \hat{a}+\frac{1}{2}\right) \hbar \omega_{\mathrm{c}}\right] U_{\mathrm{pzw}} \rightarrow\left(\hat{a}^{\dagger} \hat{a}+\frac{1}{2}\right) \hbar \omega_{\mathrm{c}}+i \sqrt{\frac{\hbar \omega_{\mathrm{c}}}{2 \varepsilon_{0} \mathcal{V}}} \hat{\epsilon} \cdot \hat{\mu}(\mathbf{r})\left(\hat{a}^{\dagger}-\hat{a}\right)+\frac{1}{\hbar \omega_{\mathrm{c}}}\left(\sqrt{\frac{\hbar \omega_{\mathrm{c}}}{2 \varepsilon_{0} \mathcal{V}}} \hat{\boldsymbol{\epsilon}} \cdot \hat{\mu}(\mathbf{r})\right)^{2}$

Note that the dipole self-energy arises from the PZW transform of the $\left(\hat{a}^{\dagger} \hat{a}+\frac{1}{2}\right) \hbar \omega_{\mathrm{c}}$ term in Eqn. S68, and is not equivalent to the $A^{2}$ term in Eqn. S62. These results can also be found in recent literature. ${ }^{4,17}$

Using the above results, the PZW transformation of the $\hat{H}_{\mathrm{p} \cdot \mathrm{A}}$ Hamiltonian (Eqn. S62) becomes

$$
\begin{aligned}
& \hat{H}_{\mathrm{d} \cdot \mathrm{E}}=U_{\mathrm{pzw}}^{\dagger} \hat{H}_{\mathrm{p} \cdot \mathrm{A}} U_{\mathrm{pzw}} \\
& =\sum_{i}^{N} \frac{p_{i}^{2}}{2 m_{i}}+V_{\mathrm{coul}}(\mathbf{r})+\left(\hat{a}^{\dagger} \hat{a}+\frac{1}{2}\right) \hbar \omega_{\mathrm{c}}+i \sqrt{\frac{\hbar \omega_{\mathrm{c}}}{2 \varepsilon_{0} \mathcal{V}}} \hat{\boldsymbol{\epsilon}} \cdot \hat{\boldsymbol{\mu}}(\mathbf{r})\left(\hat{a}^{\dagger}-\hat{a}\right)+\frac{1}{\hbar \omega_{\mathrm{c}}}\left(\sqrt{\frac{\hbar \omega_{\mathrm{c}}}{2 \varepsilon_{0} \mathcal{V}}} \hat{\boldsymbol{\epsilon}} \cdot \hat{\boldsymbol{\mu}}(\mathbf{r})\right)^{2}
\end{aligned}
$$

The above Hamiltonian, $\hat{H}_{\mathrm{d} \cdot \mathrm{E}}$ is known as the "d $\cdot \mathrm{E}$ " Hamiltonian, since the light-matter coupling term involves the dipole operator and the transverse electric field, i.e., $i \sqrt{\frac{\hbar \omega_{c}}{2 \epsilon_{0} V_{c}}} \hat{\boldsymbol{\epsilon}}$. $\hat{\boldsymbol{\mu}}(\mathbf{r})\left(\hat{a}^{\dagger}-\hat{a}\right)=-\hat{\boldsymbol{\mu}}(\mathbf{r}) \cdot \mathbf{E}_{\perp}$, where $\mathbf{E}_{\perp}=i \sqrt{\frac{\hbar \omega_{c}}{2 \epsilon_{0} V_{c}}} \hat{\boldsymbol{\epsilon}} \cdot \hat{\boldsymbol{\mu}}(\mathbf{r})\left(\hat{a}-\hat{a}^{\dagger}\right)$ is the transverse component of the $E$ filed associated with the single mode in the cavity under the long-wavelength limit.

Further, the above Hamiltonian with the complex light-matter coupling can be transformed to new Hamiltonian with a real light-matter coupling, through a phase transformation with the following unitary operator

$$
\hat{U}_{\phi}=\exp \left[i \frac{\pi}{2} \hat{a}^{\dagger} \hat{a}\right]
$$


which gives $\hat{U}_{\phi}^{\dagger} \hat{a} \hat{U}_{\phi} \rightarrow i \hat{a}$ and $\hat{U}_{\phi}^{\dagger} \hat{a}^{\dagger} \hat{U}_{\phi} \rightarrow-i \hat{a}^{\dagger}$. Applying this unitary transformation on $\hat{H}_{\mathrm{d} \cdot \mathrm{E}}$ (and using the Baker-Hausdorff identity), we obtain the following Pauli-Fierz Hamiltonian

$$
\begin{aligned}
& \hat{H}_{\mathrm{PF}}=\hat{U}_{\phi}^{\dagger} \hat{H}_{\mathrm{d} \cdot \mathrm{E}} \hat{U}_{\phi} \\
& =\sum_{i}^{N} \frac{p_{i}^{2}}{2 m_{i}}+V_{\mathrm{coul}}(\mathbf{r})+\left(\hat{a}^{\dagger} \hat{a}+\frac{1}{2}\right) \hbar \omega_{\mathrm{c}}+\sqrt{\frac{\hbar \omega_{\mathrm{c}}}{2 \epsilon_{0} V_{c}}} \hat{\boldsymbol{\epsilon}} \cdot \hat{\boldsymbol{\mu}}(\mathbf{r})\left(\hat{a}^{\dagger}+\hat{a}\right)+\frac{1}{\hbar \omega_{\mathrm{c}}}\left(\sqrt{\frac{\hbar \omega_{\mathrm{c}}}{2 \epsilon_{0} V_{c}}} \hat{\boldsymbol{\epsilon}} \cdot \hat{\boldsymbol{\mu}}(\mathbf{r})\right)^{2} .
\end{aligned}
$$

Here, $\sum_{i}^{N} \frac{p_{i}^{2}}{2 m_{i}}+V_{\text {coul }}(\mathbf{r}) \equiv \hat{H}_{\mathrm{M}}$ represents the molecular Hamiltonian, which includes all electrons and nuclei. $\hat{H}_{\mathrm{PF}}$ is the Hamiltonian we used in main text.

\section{References}

(1) Pacher, T.; Mead, C. A.; Cederbaum, L. S.; Koppel, H. Gauge Theory and Quasidiabatic States in Molecular Physics. J. Chem. Phys. 1989, 91, 7057.

(2) Flick, J.; Ruggenthaler, M.; Appel, H.; Rubio, A. Atoms and Molecules in Cavities, from Weak to Strong Coupling in Quantum-Electrodynamics (QED) Chemistry. Proc. Natl. Acad. Sci. U. S. A. 2017, 114, 3026-3034.

(3) Stefano, O. D.; Settineri, A.; Macri, V.; Garziano, L.; Stassi, R.; Savasta, S.; Nori, F. Resolution of Gauge Ambiguities in Ultrastrong-Coupling Cavity Quantum Electrodynamics. Nature Phys. 2019, 15, 803-808.

(4) Semenov, A.; Nitzan, A. Electron Transfer in Confined Electromagnetic Fields. J. Chem. Phys. 2019, 150, 174122.

(5) Irish, E. K.; Gea-Banacloche, J.; Martin, I.; Schwab, K. C. Dynamics of a Two-Level System Strongly Coupled to a High-Frequency Quantum Oscillator. Phys. Rev. B 2005, 72, 195410. 
(6) Irish, E. K. Generalized Rotating-Wave Approximation for Arbitrarily Large Coupling. Phys. Rev. Lett. 2007, 99, 173601.

(7) Marston, C. C.; Balint-Kurti, G. G. The Fourier Grid Hamiltonian Method for Bound State Eigenvalues and Eigenfunctions. J. Chem. Phys. 1989, 91, 3571-3576.

(8) Tannor, D. Introduction to Quantum Mechanics: A Time-Dependent Perspective. University Science books: Mill Valley, U.S.A 2007,

(9) Mulliken, R. S. Molecular Compounds and their Spectra. II. J. Am. Chem. Soc. 1952, $74,811-824$.

(10) Cave, R. J.; Newton, M. D. Generalization of the Mulliken-Hush Treatment for the Calculation of Electron Transfer Matrix Elements. Chem. Phys. Lett. 1996, 249, $15-$ 19.

(11) Cave, R. J.; Newton, M. D. Calculation of Electronic Coupling Matrix Elements for Ground and Excited State Electron Transfer Reactions: Comparison of the Generalized Mulliken-Hush and Block Diagonalization Methods. J. Chem. Phys. 1997, 106, 92139226.

(12) Hush, N. S. Intervalence-Transfer Absorption. Part 2. Theoretical Considerations and Spectroscopic Data. John Wiley \& Sons, Inc.: Hoboken, U.S.A. 2007,

(13) Giese, T. J.; York, D. M. Complete Basis Set Extrapolated Potential Energy, Dipole, and Polarizability Surfaces of Alkali Halide Ion-Neutral Weakly Avoided Crossings With and Without Applied Electric Fields. J. Chem. Phys. 2004, 120, 7939-7948.

(14) Schäfer, C.; Ruggenthaler, M.; Rubio, A. Ab Initio Nonrelativistic Quantum Electrodynamics: Bridging Quantum Chemistry and Quantum Optics From Weak to Strong Coupling. Phys. Rev. A 2018, 98, 043801. 
(15) Taylor, M. A. D.; Mandal, A.; Zhou, W.; Huo, P. Resolution of Gauge Ambiguities in Molecular Cavity Quantum Electrodynamics. Phys. Rev. Lett. 2020, 125, 123602.

(16) Bernardis, D. D.; Pilar, P.; Jaako, T.; Liberato, S. D.; Rabl, P. Breakdown of Gauge Invariance in Ultrastrong-Coupling Cavity QED. Phys. Rev. A 2018, 98, 053819.

(17) Rokaj, V.; Welakuh, D. M.; Ruggenthaler, M.; Rubio, A. Light-Matter Interaction in the Longwavelength Limit: No Ground-State Without Dipole Self-Energy. J. Phys. B: At. Mol. Opt. Phys. 2018, 51, 034005.

(18) Grynberg, G.; Aspect, A.; Fabre, C. Introduction to Quantum Optics: From the Semiclassical Approach to Quantized Light. Cambridge University Press: Cambridge, U.K. 2010

(19) Cohen-Tannoudji, C.; Dupont-Roc, J.; Grynberg, G. Photons and Atoms: Introduction to Quantum Electrodynamics. John Wiley 6 Sons, Inc.: Hoboken, U.S.A. 1989, 